\title{
HOMOCLINIC LOOP AND MULTIPLE LIMIT CYCLE BIFURCATION SURFACES
}

\author{
L. M. PERKO
}

\begin{abstract}
This paper establishes the existence and analyticity of homoclinic loop bifurcation surfaces $\mathscr{H}$ and multiplicity-two, limit cycle bifurcation surfaces $\mathscr{C}$ for planar systems depending on two or more parameters; it determines the side of $\mathscr{H}$ or $\mathscr{C}$ on which limit cycles occur; and it shows that if $\mathscr{H}$ and $\mathscr{C}$ intersect, then typically they do so at a flat contact.
\end{abstract}

\section{INTRODUCTION}

Consider a $C^{k}$ or analytic system

$$
\dot{\mathbf{x}}=\mathbf{f}(\mathbf{x}, \boldsymbol{\mu}),
$$

with $1 \leq k \leq \infty, \mathbf{x} \in \mathbf{R}^{2}, \mu \in \mathbf{R}^{m}$, and $m \geq 2$, which has a homoclinic orbit $\gamma_{0}(t)$ or a semistable, multiplicity-two limit cycle $\mathbf{x}_{0}(t)$ for $\boldsymbol{\mu}=\boldsymbol{\mu}_{0}$. Typically, as $\mu$ varies from $\mu_{0}$, the homoclinic loop breaks or the semistable limit cycle either vanishes or splits into two simple limit cycles. However, if certain integrals along $\gamma_{0}(t)$ or $\mathbf{x}_{0}(t)$ are not equal to zero, then it is shown that there are $(m-1)$-dimensional, $C^{k}$ or analytic surfaces $\mathscr{H}$ or $\mathscr{C}$ through the point $\boldsymbol{\mu}_{0}$ in the parameter space $\mathbf{R}^{m}$ on which $\left(1_{\mu}\right)$ has a homoclinic loop or a semistable, multiplicity-two limit cycle (if $k \geq 2$ in the latter case); furthermore, the signs of these integrals determine the side of $\mathscr{H}$ on which a limit cycle bifurcates from the homoclinic loop $\gamma_{0}(t)$ and the side of $\mathscr{C}$ on which two simple limit cycles bifurcate from the multiplicity-two limit cycle $\mathbf{x}_{0}(t)$. If the surfaces $\mathscr{H}$ and $\mathscr{C}$ intersect, then for $C^{\infty}$ or analytic systems it is shown that they typically do so at a flat contact.

If for $\boldsymbol{\mu}=\mu_{0}$, the system $\left(1_{\mu}\right)$ has a multiple limit cycle $\mathbf{x}_{0}(t)$ of multiplicitytwo, then a classical formula for the derivative of the Poincare map or of the displacement function with respect to the parameter $\boldsymbol{\mu}$, equation (36) on p. 384 in [1], makes it possible to use the Weierstrass preparation theorem to establish the existence of a multiplicity-two, limit cycle bifurcation surface $\mathscr{C}$ for $\left(1_{\mu}\right)$ provided that certain integrals along $\mathbf{x}_{0}(t)$ are not all zero and $k \geq 2$. This formula has proved to be immensely useful in studying bifurcations of limit cycles; cf. [2-6]. On the other hand, if $\left(1_{\mu}\right)$ has a homoclinic orbit $\gamma_{0}(t)$ for $\mu=\mu_{0}$, a formula for the derivative of the displacement function with respect to the parameter $\boldsymbol{\mu}$ is not readily available in the literature. (However,

Received by the editors January 31, 1992.

1991 Mathematics Subject Classification. Primary 58F14; Secondary 58F21. 
integral formulas for the distance between saddle separatrices do appear in the literature and even in some of Poincaré's early work; $\mathrm{cf}$. the historical comments in Remark 11 at the end of Appendix I.) Thus, in $\S 2$, we define the displacement function $d(\boldsymbol{\mu})$ which measures the distance between the saddle separatrices of $\left(1_{\mu}\right)$ that form the homoclinic loop $\gamma_{0}(t)$ for $\boldsymbol{\mu}=\boldsymbol{\mu}_{0}$, establish that it is a $C^{k}$ or analytic function of $\mu$, and then employ a technique used by Duff [7] in the early 1950 s for integrating the first variation of $\left(1_{\mu}\right)$ with respect to $\boldsymbol{\mu}$ along a trajectory of $\left(1_{\mu}\right)$ in order to derive a formula for $d_{\mu}\left(\mu_{0}\right)$ in terms of certain integrals along $\gamma_{0}(t)$; cf. Lemma 2 in $\S 2$ of this paper. These integrals are related to the Melnikov integral for perturbed dynamical systems introduced by Melnikov in the early 1960s; cf. [8] or [9]. This relationship is discussed in Appendix I. The formula for $d_{\mu}\left(\mu_{0}\right)$ then makes it possible to use the implicit function theorem to establish the existence of a homoclinic loop bifurcation surface $\mathscr{H}$ for $\left(1_{\mu}\right)$ provided that certain integrals along $\gamma_{0}(t)$ are not all zero. This technique for establishing the existence (and analyticity) of the homoclinic loop bifurcation surface $\mathscr{H}$ for $\left(1_{\mu}\right)$ generalizes the technique used by the author in [10] to analyze the Bogdanov-Takens system. Several interesting examples of systems with homoclinic loop and/or multiple limit cycle bifurcation surfaces appear in the literature; cf., e.g., [10-15]. Some of the results in [10-15] follow directly from the theorems in this paper and, in addition, it follows from Theorem 5 and its corollary in $\S 4$ that the surfaces $\mathscr{H}$ and $\mathscr{C}$ for the systems in [11-15] intersect at a flat contact. The nature of the intersection of the homoclinic loop bifurcation curve $\mathscr{H}$ and the multiplicitytwo, limit cycle bifurcation curve $\mathscr{C}$ was not discussed in [12] or [13]; however, their intersection was shown to be exponentially flat for the specific systems studied in $[11,14$, and 15] and this result was recently generalized to generic systems of the form $\left(1_{\mu}\right)$ with $\mathbf{x} \in \mathbf{R}^{n}, \boldsymbol{\mu} \in \mathbf{R}^{2}$ and $7 \leq k \leq \infty$ in [16]. We extend this result in $\S 4$ where we show that $\mathscr{H}$ and $\mathscr{C}$ intersect at a flat contact for generic systems $\left(1_{\mu}\right)$ with $\mathbf{x} \in \mathbf{R}^{2}, \boldsymbol{\mu} \in \mathbf{R}^{2}$, and $k=\infty$. Note that the intersection of two curves being exponentially flat does not necessarily imply that they intersect at a flat contact. For example, the function

$$
\kappa(x)=e^{-1 / x} \sin \left(e^{1 / x}\right)
$$

is exponentially flat at $x=0$, but it does not have a flat contact, i.e., a contact of infinite order, with the $x$-axis at $x=0$ since $\kappa^{\prime}\left(0^{+}\right)$does not exist. These concepts are defined and discussed more thoroughly in $\S 4$.

We begin this paper with a discussion of homoclinic loop and multiplicitytwo, limit cycle bifurcation surfaces in $\S \S 2$ and 3 respectively and then discuss their intersections in $\S 4$.

\section{HOMOCLINIC LOOP BIFURCATION SURFACES}

Suppose that the $C^{k}$ or analytic system $\left(1_{\mu}\right)$, with $1 \leq k \leq \infty, \mathbf{x} \in \mathbf{R}^{2}$, $\boldsymbol{\mu} \in \mathbf{R}^{m}$, and $m \geq 2$, has a homoclinic loop

$$
\Gamma_{0}: \mathbf{x}=\gamma_{0}(t), \quad-\infty<t<\infty,
$$

at a hyperbolic saddle $\mathbf{0}$ for $\boldsymbol{\mu}=\boldsymbol{\mu}_{0}$. Then $S_{0}=\Gamma_{0} \cup\{\boldsymbol{0}\}$ is a separatrix cycle and typically, as $\boldsymbol{\mu}$ varies from $\mu_{0}$ in the parameter space $\mathbf{R}^{m}, S_{0}$ vanishes, 
i.e., the saddle connection is broken. However, if one of the integrals

$$
I_{j}=\int_{-\infty}^{\infty} e^{-\int_{0}^{t} \nabla \cdot \mathbf{f}\left(\gamma_{0}(s), \mu_{0}\right) d s} \mathbf{f} \wedge \mathbf{f}_{\mu_{j}}\left(\gamma_{0}(t), \boldsymbol{\mu}_{0}\right) d t
$$

for $j=1, \ldots, m$, does not vanish, then locally there is an $(m-1)$ dimensional, $C^{k}$ or analytic manifold $\mathscr{H}$ on which $\left(1_{\mu}\right)$ has a homoclinic loop. This is made precise in the following theorem where we assume that $I_{1} \neq 0$ and let $\boldsymbol{\mu}_{0}=\left(\mu_{1}^{(0)}, \ldots, \mu_{m}^{(0)}\right)$.

Theorem 1. Suppose that for $\boldsymbol{\mu}=\mu_{0}$ the $C^{k}$ or analytic system $\left(1_{\mu}\right)$ with $1 \leq k \leq \infty$ and $m \geq 2$ has a homoclinic orbit $\gamma_{0}(t)$ at a hyperbolic saddle of $\left(1_{\mu_{0}}\right)$ and that $I_{1} \neq 0$. Then given $\varepsilon>0$, there exists $a \delta>0$ and $a$ unique function $h\left(\mu_{2}, \ldots, \mu_{m}\right)$ with $h\left(\mu_{2}^{(0)}, \ldots, \mu_{m}^{(0)}\right)=\mu_{1}^{(0)}$, defined and $C^{k}$ or analytic for $\left|\mu_{j}-\mu_{j}^{(0)}\right|<\delta, j=2, \ldots, m$, such that the system $\left(1_{\mu}\right)$ with $\mu_{1}=h\left(\mu_{2}, \ldots, \mu_{m}\right)$ and $\left.\mid \mu_{j}-\mu_{j}^{(0)}\right) \mid<\delta$ for $j=2, \ldots, m$ has a unique homoclinic orbit in an $\varepsilon$-neighborhood of $\Gamma_{0}$; i.e., $\left(1_{\mu}\right)$ has a unique, local, ( $m-$ 1) dimensional homoclinic loop bifurcation surface $\mathscr{H}: \mu_{1}=h\left(\mu_{2}, \ldots, \mu_{m}\right)$ through the point $\boldsymbol{\mu}_{0} \in \mathbf{R}^{m}$.

Before proving this theorem, we state two important corollaries which determine the side of the homoclinic loop bifurcation surface $\mathscr{H}$ on which a limit cycle bifurcates from the homoclinic loop of $\left(1_{\mu}\right)$ with $\mu_{1}=h\left(\mu_{2}, \ldots, \mu_{m}\right)$. In order to succinctly state these corollaries, we let $\omega_{0}= \pm 1$ according to whether the separatrix cycle $S_{0}$ is positively or negatively oriented respectively and we note that if $\left(1_{\mu_{0}}\right)$ has a homoclinic loop $\Gamma_{0}$ at a hyperbolic saddle at 0 then according to Lemma 2 on p. 300 in [1], the Poincare map for $\left(1_{\mu_{0}}\right)$ is defined along a normal line to $\Gamma_{0}$ either on the interior or on the exterior of $S_{0}=\Gamma_{0} \cup\{\boldsymbol{0}\}$; cf. Figure 1 (a) or (b) respectively (see next page).

In view of the above observation, if $\nabla \cdot \mathbf{f}\left(\mathbf{0}, \mu_{0}\right) \neq 0$ we can define the quantity

$$
\boldsymbol{\sigma}_{0} \equiv \pm \operatorname{sgn}\left[\nabla \cdot \mathbf{f}\left(\mathbf{0}, \boldsymbol{\mu}_{0}\right)\right]
$$

according to whether the Poincare map is defined on the exterior or on the interior of $S_{0}$ respectively. It then follows from Theorem 44 on p. 304 in [1] or from Theorem 1 in $\S 4.7$ of [9] that if $\nabla \cdot \mathbf{f}\left(\mathbf{0}, \boldsymbol{\mu}_{0}\right) \neq 0$ and the Poincaré map is defined on the exterior of $S_{0}$ then $S_{0}$ is unstable or stable on its exterior iff $\sigma_{0}= \pm 1$ respectively; and if $\nabla \cdot \mathbf{f}\left(\mathbf{0}, \mu_{0}\right) \neq 0$ and the Poincare map is defined on the interior of $S_{0}$ then $S_{0}$ is unstable or stable on its interior iff $\sigma_{0}=\mp 1$ respectively.

Corollary 1. Suppose that in addition to the hypotheses of Theorem $1, \nabla \cdot \mathbf{f}\left(\mathbf{0}, \boldsymbol{\mu}_{0}\right)$ $\neq 0$ and we let $\sigma_{0}$ be the quantity defined by (3). If we let $\Delta \mu_{1}=\mu_{1}-$ $h\left(\mu_{2}, \ldots, \mu_{m}\right)$, then given $\varepsilon>0$ there exists a $\delta>0$ such that for $\left|\boldsymbol{\mu}-\mu_{0}\right|<\boldsymbol{\delta}$

(a) the system $\left(1_{\mu}\right)$ has a unique hyperbolic limit cycle in an $\varepsilon$-neighborhood of $\Gamma_{0}$ if $\omega_{0} \sigma_{0} I_{1} \Delta \mu_{1}>0$,

(b) the system $\left(1_{\mu}\right)$ has a unique separatrix cycle in an $\varepsilon$-neighborhood of $\Gamma_{0}$ iff $\Delta \mu_{1}=0$, and

(c) the system $\left(1_{\mu}\right)$ has no limit cycle or separatrix cycle in an $\varepsilon$-neighborhooa of $\Gamma_{0}$ if $\omega_{0} \sigma_{0} I_{1} \Delta \mu_{1}<0 ;$;f. Figure 2 . 


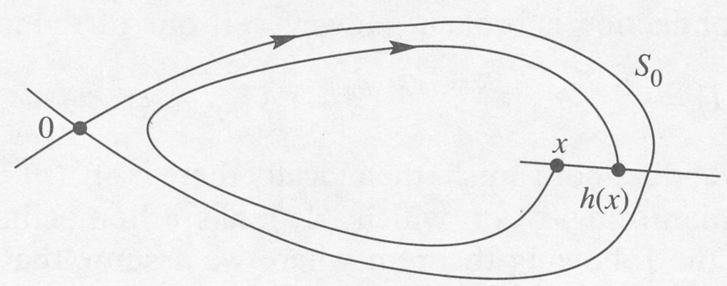

(a)

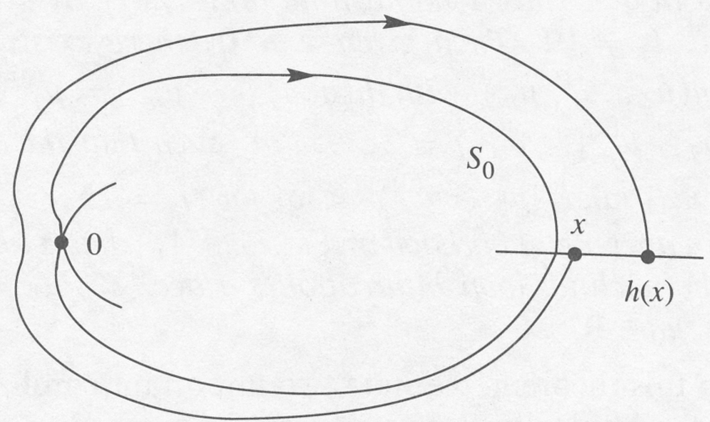

(b)

FIGURE 1. The Poincare map, $h(x)$, for $\left(1_{\mu_{0}}\right)$ is defined either on (a) an interior neighborhood of $S_{0}$ or on (b) an exterior neighborhood of $S_{0}$

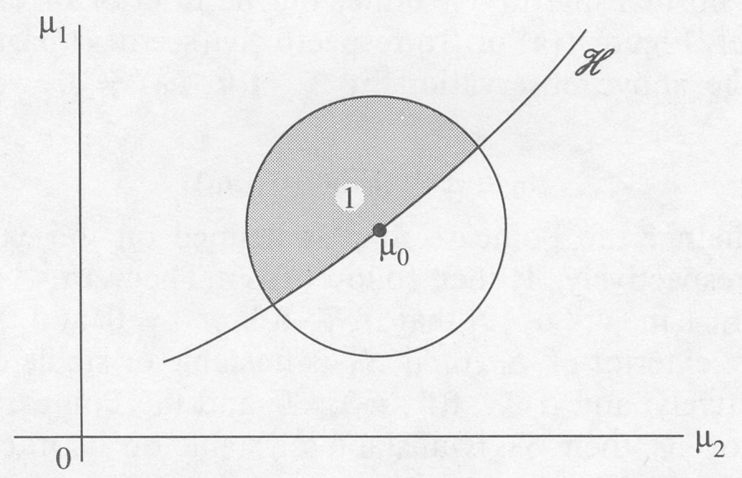

FIGURE 2. If $\omega_{0} \sigma_{0} I_{1}>0$, then locally $\left(1_{\mu}\right)$ has a unique limit cycle near $\Gamma_{0}$ if $\mu_{1}>h\left(\mu_{2}, \ldots, \mu_{m}\right)$ and no limit cycle near $\Gamma_{0}$ if $\mu_{1}<h\left(\mu_{2}, \ldots, \mu_{m}\right)$

Next, let us define the quantity

$$
I_{0} \equiv \pm \int_{-\infty}^{\infty} \nabla \cdot \mathbf{f}\left(\gamma_{0}(t), \boldsymbol{\mu}_{0}\right) d t
$$

according to whether the Poincare map is defined on the exterior or on the interior of $S_{0}$ respectively; cf. Figure 1. If $\nabla \cdot \mathbf{f}\left(\mathbf{0}, \boldsymbol{\mu}_{0}\right) \neq 0$ then according to Lemma 6 on p. 302 of [1], $I_{0}= \pm \infty$; however, if $\nabla \cdot \mathbf{f}\left(\mathbf{0}, \boldsymbol{\mu}_{0}\right)=0$, then the quantity $I_{0}$ may exist as a nonzero real number. If so then it follows from 
Lemma 5 on p. 293 in [1] and equation (5) on p. 301 in [1] that $S_{0}$ is unstable or stable on its exterior if $I_{0}$ is positive or negative respectively and that $S_{0}$ is unstable or stable on its interior if $I_{0}$ is negative or positive respectively.

Corollary 2. Under the hypotheses of Theorem 1 , if the quantity $I_{0}$ defined by equation (4) is a nonzero, real number, then given $\varepsilon>0$, there exists $a \delta>0$ such that for $\left|\boldsymbol{\mu}-\boldsymbol{\mu}_{0}\right|<\delta$ and $\Delta \mu_{1} \equiv \mu_{1}-h\left(\mu_{2}, \ldots, \mu_{m}\right)$,

(a) the system $\left(1_{\mu}\right)$ has a unique hyperbolic limit cycle in an $\varepsilon$-neighborhood of $\Gamma_{0}$ if $\omega_{0} I_{0} I_{1} \Delta \mu_{1}>0$,

(b) the system $\left(1_{\mu}\right)$ has a unique separatrix cycle in an $\varepsilon$-neighborhood of $\Gamma_{0}$ iff $\Delta \mu_{1}=0$, and

(c) the system $\left(1_{\mu}\right)$ either has exactly two hyperbolic limit cycles, or a unique multiplicity-two limit cycle, or no limit cycle in an $\varepsilon$-neighborhood of $\Gamma_{0}$ if $\omega_{0} I_{0} I_{1} \Delta \mu_{1}<0$.

Remark 1 . If the system $\left(1_{\mu}\right)$ defines a one-parameter family of rotated vector fields with parameter $\mu_{1}$, then according to the definition of a family of rotated vector fields, Definition 1 in [7], $\mathbf{f} \wedge \mathbf{f}_{\mu_{1}}>0$ and it follows that $I_{1}>0$. Hence, all of the above results hold and, furthermore, in the case when $\left(1_{\mu}\right)$ is an analytic system, the rotated vector field theory in [7 and 17] and the uniqueness of analytic continuations, Theorems 4-6, p. 34 in [18], allow us to establish the existence of a global, analytic, homoclinic loop bifurcation surface $\mathscr{H}$. This was carried out for the Bogdanov-Takens system

$$
\dot{x}=y, \quad \dot{y}=x(x-1)+\mu_{1} y+\mu_{2} x y
$$

in [10]; cf. Figure 3. Note that it follows immediately from the above differential equations for the Bogdanov-Takens system that

$$
\omega_{0}=1, \quad \sigma_{0} \equiv-\operatorname{sgn}[\nabla \cdot \mathbf{f}(\mathbf{0}, \mathbf{0})]=-\mu_{1},
$$

and that $I_{1}>0$ since the Bogdanov-Takens system defines a one-parameter family of (positively) rotated vector fields with parameter $\mu_{1}$. The side of the homoclinic loop bifurcation surface $\mathscr{H}$ on which the Bogdanov-Takens system has a unique limit cycle therefore follows from Corollary 1; cf. Figure 3.

The fact that there exists a unique limit cycle in the global region shaded in Figure 3 follows from the uniqueness of the limit cycle of the BogdanovTakens system, established in [19], and the theory of rotated vector fields in

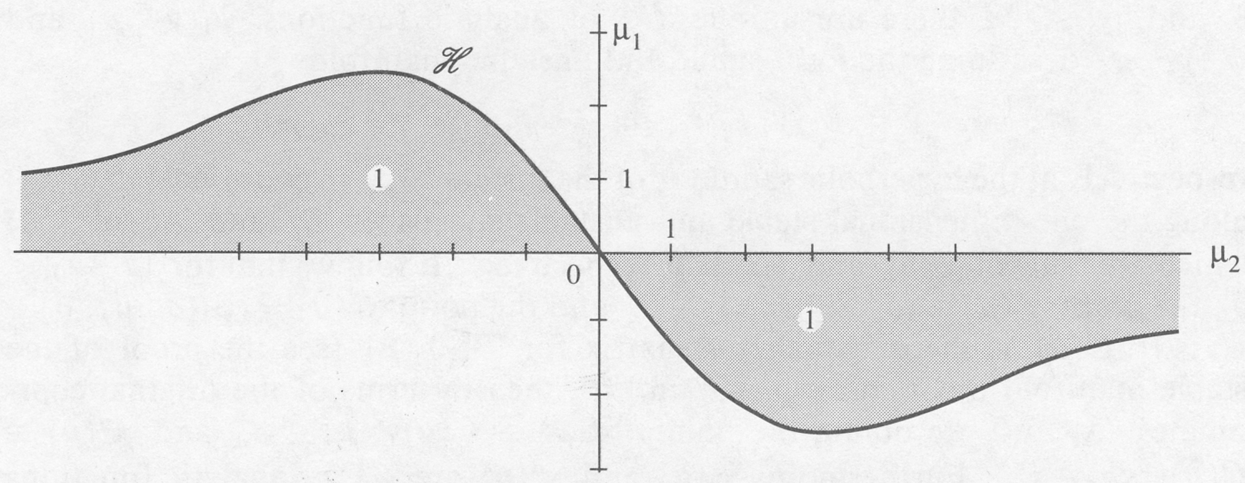

FIGURE 3. The global homoclinic loop bifurcation curve $\mathscr{H}$ for the Bogdanov-Takens system 
[17] which shows that the Bogdanov-Takens system has a supercritical (or subcritical) Hopf bifurcation at $\mu_{1}=0$ if $\mu_{2}<0$ (or if $\mu_{2}>0$ ). The shape of the global, homoclinic loop bifurcation curve $\mathscr{H}$ shown in Figure 3 was determined numerically.

Remark 2. If the separatrix cycle $S_{0}$ is the boundary of a period annulus of $\left(1_{\mu_{0}}\right)$, i.e., the boundary of a one-parameter family of periodic orbits of $\left(1_{\mu_{0}}\right)$, then both $\nabla \cdot \mathbf{f}\left(\mathbf{0}, \boldsymbol{\mu}_{0}\right)=0$ and the integral defined in (3), $I_{0}=0$. However, even in this highly degenerate case, some results determining the side of $\mathscr{H}$ on which a limit cycle bifurcates from the homoclinic loop $\Gamma_{0}$ of $\left(1_{\mu_{0}}\right)$ are available for perturbed periodic systems when $I_{1} \neq 0$. See Theorems 3.3 and 3.5 in [6] or Theorem 3 in [20].

In order to prove Theorem 1 , we first note that if the system $\left(1_{\mu_{0}}\right)$ has a hyperbolic saddle at $\mathbf{0}$, then $\operatorname{det} D \mathbf{f}\left(\mathbf{0}, \boldsymbol{\mu}_{0}\right)<0$ and it follows from Theorems 1 and 2, p. 305 in [21] that for sufficiently small $\left|\boldsymbol{\mu}-\boldsymbol{\mu}_{0}\right|$, the system $\left(1_{\mu}\right)$ has a hyperbolic saddle at a point $\mathbf{x}(\boldsymbol{\mu})$ which approaches $\mathbf{0}$ as $\boldsymbol{\mu} \rightarrow \boldsymbol{\mu}_{0}$; in fact, $\mathbf{x}(\boldsymbol{\mu})$ is a $C^{k}$ or analytic function for all sufficiently small $\left|\boldsymbol{\mu}-\boldsymbol{\mu}_{0}\right|$. Therefore, under the $C^{k}$ or analytic change of coordinates $\mathbf{x} \rightarrow \mathbf{x}-\mathbf{x}(\boldsymbol{\mu})$, the system $\left(1_{\boldsymbol{\mu}}\right)$ will have a hyperbolic saddle at the origin for sufficiently small $\left|\boldsymbol{\mu}-\boldsymbol{\mu}_{0}\right|$ and this is assumed to hold throughout the remainder of this paper.

We let $\mathbf{x}_{0}=\gamma_{0}(\mathbf{0})$ and define the unit vector $\mathbf{u}_{0}=\mathbf{f}\left(\mathbf{x}_{0}, \boldsymbol{\mu}_{0}\right) /\left|\mathbf{f}\left(\mathbf{x}_{0}, \boldsymbol{\mu}_{0}\right)\right|$, and the normal line

$$
l_{0}=\left\{\mathbf{x} \in \mathbf{R}^{2} \mid\left(\mathbf{x}-\mathbf{x}_{0}\right) \cdot \mathbf{f}\left(\mathbf{x}_{0}, \boldsymbol{\mu}_{0}\right)=0\right\} .
$$

See Figure 4. As was mentioned in the introduction, Theorem 1 follows from the implicit function theorem by using a formula for the derivative of the displacement function $d(\boldsymbol{\mu})$ with respect to $\boldsymbol{\mu}$. So in order to prove Theorem 1, we first of all define the displacement function $d(\boldsymbol{\mu})$, establish its continuity or analyticity, and derive a formula for $d_{\mu}\left(\mu_{0}\right)$. Geometrically, the displacement function $d(\boldsymbol{\mu})$ is the distance between the saddle separatrices $\Gamma_{\mu}^{s}$ and $\Gamma_{\mu}^{u}$ along the normal $l_{0}$ to the homoclinic loop $\Gamma_{0}$ as shown in Figure 4. Clearly $d\left(\boldsymbol{\mu}_{0}\right)=0$.

In order to give a precise analytical definition for $d(\mu)$ and to establish that it is a $C^{k}$ or analytic function, we use the stable manifold theorem, Theorem 1.3.1, p. 13 in [8] or Theorem 1, p. 107 in [9] (cf. Theorem 1 in Appendix II of [10] for the analytic case). Under the hypotheses of Theorem 1, the stable manifold theorem implies that there exists a $\delta>0$ such that for $\left|\boldsymbol{\mu}-\boldsymbol{\mu}_{0}\right|<$ $\delta$ and $|\mathbf{y}|<2 \delta$ there are unique $C^{k}$ or analytic functions $\psi_{1}\left(y_{2}, \boldsymbol{\mu}\right)$ and $\psi_{2}\left(y_{1}, \boldsymbol{\mu}\right)$ describing the local stable and unstable manifolds

$$
S_{\boldsymbol{\mu}}: y_{2}=\psi_{2}\left(y_{1}, \boldsymbol{\mu}\right) \text { and } U_{\boldsymbol{\mu}}: y_{1}=\psi_{1}\left(y_{2}, \boldsymbol{\mu}\right)
$$

respectively at the hyperbolic saddle 0 of the system $\left(1_{\mu}\right)$ in coordinates $\left(y_{1}, y_{2}\right)$ along the one-dimensional stable and unstable subspaces $E_{\mu}^{s}$ and $E_{\mu}^{u}$ of $\left(1_{\mu}\right)$ which are tangent to $S_{\mu}$ and $U_{\mu}$ at 0 respectively. It follows that for $\left|\boldsymbol{\mu}-\boldsymbol{\mu}_{0}\right|<$ $\delta$, the point $\mathbf{y}^{s}(\boldsymbol{\mu}) \equiv\left(\delta, \psi_{2}(\delta, \boldsymbol{\mu})\right) \in S_{\mu}$ and the point $\mathbf{y}^{u}(\boldsymbol{\mu})=\left(\psi_{1}(\boldsymbol{\delta}, \boldsymbol{\mu}), \delta\right) \in$ $U_{\boldsymbol{\mu}}$. If $C(\boldsymbol{\mu})$ is the diagonalizing matrix for $D \mathbf{f}(\mathbf{0}, \boldsymbol{\mu})$ (see the proof of the stable manifold theorem on p. 107 in [9]), then in terms of the original coordinates $\left(x_{1}, x_{2}\right)$ we obtain the points $\mathbf{x}^{s}(\boldsymbol{\mu}) \equiv C(\boldsymbol{\mu}) \mathbf{y}^{s}(\boldsymbol{\mu}) \in S_{\boldsymbol{\mu}}$ and $\mathbf{x}^{u}(\boldsymbol{\mu}) \equiv$ $C(\boldsymbol{\mu}) \mathbf{y}^{u}(\boldsymbol{\mu}) \in U_{\boldsymbol{\mu}}$. Furthermore, $\mathbf{x}^{s}(\boldsymbol{\mu})$ and $\mathbf{x}^{u}(\boldsymbol{\mu})$ are $C^{k}$ or analytic functions of $\boldsymbol{\mu}$ for $\left|\boldsymbol{\mu}-\boldsymbol{\mu}_{0}\right|<\delta$. 

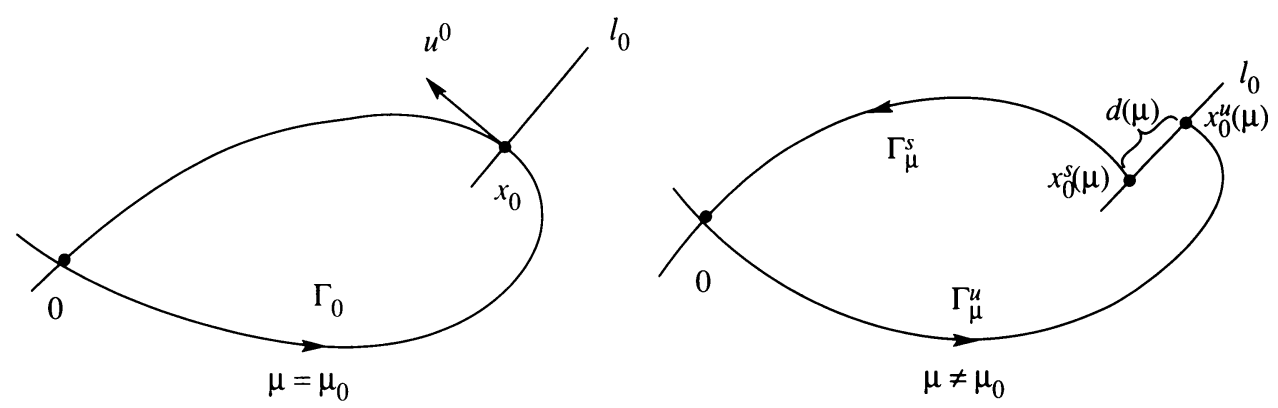

FIgURE 4. The displacement function $d(\boldsymbol{\mu})$ defined for $\boldsymbol{\mu}$ near $\boldsymbol{\mu}_{0}$

Now let $\phi(t, \xi, \mu)$ denote the solution of $\left(1_{\mu}\right)$ which satisfies the initial condition $\phi(0, \boldsymbol{\xi}, \boldsymbol{\mu})=\boldsymbol{\xi}$. We can then define the following $C^{k}$ or analytic functions representing the separatrices $\Gamma_{\mu}^{s}$ and $\Gamma_{\mu}^{u}$ respectively: $\mathbf{x}^{s}(t, \boldsymbol{\mu}) \equiv$ $\phi\left(t, \mathbf{x}^{s}(\boldsymbol{\mu}), \boldsymbol{\mu}\right)$ and $\mathbf{x}^{u}(t, \boldsymbol{\mu}) \equiv \phi\left(t, \mathbf{x}^{u}(\boldsymbol{\mu}), \boldsymbol{\mu}\right)$ for $\left|\boldsymbol{\mu}-\boldsymbol{\mu}_{0}\right|<\delta$ and $t \in \mathbf{R}$. Then since the homoclinic loop $\Gamma_{0}$ of $\left(1_{\mu_{0}}\right)$ crosses the normal line $l_{0}$ at $\mathbf{x}_{0}$, it follows that there exists a $t_{0}^{s}<0$ and a $t_{0}^{u}>0$ such that $\mathbf{x}^{s}\left(t_{0}^{s}, \mu_{0}\right)=\mathbf{x}_{0}=$ $\mathbf{x}^{u}\left(t_{0}^{u}, \mu_{0}\right)$. In fact, by the uniqueness of solutions, we have $\mathbf{x}^{s}\left(t+t_{0}^{s}, \mu_{0}\right)=$ $\gamma_{0}(t)=\mathbf{x}^{u}\left(t+t_{0}^{u}, \mu_{0}\right)$ for all $t \in \mathbf{R}$. We now define the points $\mathbf{x}_{0}^{s}(\boldsymbol{\mu})$ and $\mathbf{x}_{0}^{u}(\boldsymbol{\mu})$ shown in Figure 4 where the separatrices $\Gamma_{\mu}^{s}$ and $\Gamma_{\mu}^{u}$ cross the line $l_{0}$ respectively.

Lemma 1. Under the hypotheses of Theorem 1, there exists $a \delta>0$ and unique functions $\tau^{s}(\boldsymbol{\mu})$ and $\tau^{u}(\boldsymbol{\mu})$ which are $C^{k}$ or analytic for $\left|\boldsymbol{\mu}-\boldsymbol{\mu}_{0}\right|<\delta$ such that $\tau^{s}(\boldsymbol{\mu}) \rightarrow t_{0}^{s}$ and $\tau^{u}(\boldsymbol{\mu}) \rightarrow t_{0}^{u}$ as $\boldsymbol{\mu} \rightarrow \boldsymbol{\mu}_{0}$ and such that the points $\mathbf{x}_{0}^{s}(\boldsymbol{\mu}) \equiv$ $\mathbf{x}^{s}\left(\tau^{s}(\boldsymbol{\mu}), \boldsymbol{\mu}\right) \in l_{0}$ and $\mathbf{x}^{u}(\boldsymbol{\mu}) \equiv \mathbf{x}^{u}\left(\tau^{u}(\boldsymbol{\mu}), \boldsymbol{\mu}\right) \in l_{0}$ for $\left|\boldsymbol{\mu}-\boldsymbol{\mu}_{0}\right|<\delta ; c f$. Figure 4. Proof. For $t \in \mathbf{R}$ and sufficiently small $\left|\boldsymbol{\mu}-\boldsymbol{\mu}_{0}\right|$, let $\mathbf{x}^{s}(t, \boldsymbol{\mu})$ be the $C^{k}$ or analytic function, representing the separatrix $\Gamma_{\mu}^{s}$, defined above and let

$$
S(t, \mu) \equiv\left[\mathbf{x}^{s}(t, \mu)-\mathbf{x}_{0}\right] \cdot \mathbf{f}\left(\mathbf{x}_{0}, \boldsymbol{\mu}_{0}\right) .
$$

Then $S(t, \mu)$ is a $C^{k}$ or analytic function for $t \in \mathbf{R}$ and all sufficiently small $\left|\boldsymbol{\mu}-\boldsymbol{\mu}_{0}\right|$. Furthermore, $S\left(t_{0}^{s}, \boldsymbol{\mu}_{0}\right)=0$ since $\mathbf{x}^{s}\left(t_{0}^{s}, \boldsymbol{\mu}_{0}\right)=\mathbf{x}_{0}$ and

$$
\frac{\partial S}{\partial t}\left(t_{0}^{s}, \boldsymbol{\mu}_{0}\right)=\dot{\mathbf{x}}^{s}\left(t_{0}^{s}, \boldsymbol{\mu}_{0}\right) \cdot \mathbf{f}\left(\mathbf{x}_{0}, \boldsymbol{\mu}_{0}\right)=\dot{\gamma}_{0}(0) \cdot \mathbf{f}\left(\mathbf{x}_{0}, \boldsymbol{\mu}_{0}\right)=\left|\mathbf{f}\left(\mathbf{x}_{0}, \boldsymbol{\mu}_{0}\right)\right|^{2} \neq 0
$$

since $\mathbf{x}_{0}=\gamma_{0}(0)$ is not a critical point of $\left(1_{\mu_{0}}\right)$. Thus, by the implicit function theorem for $C^{k}$ or analytic functions, Theorems 10.2 .3 and 10.2.4, p. 268 in [22] respectively, there exist a $\delta>0$ and a unique function $\tau^{s}(\boldsymbol{\mu})$ which is $C^{k}$ or analytic for $\left|\boldsymbol{\mu}-\boldsymbol{\mu}_{0}\right|<\delta$ such that $\tau^{s}(\boldsymbol{\mu}) \rightarrow t_{0}^{s}$ as $\boldsymbol{\mu} \rightarrow \boldsymbol{\mu}_{0}$ and such that $S\left(\tau^{s}(\boldsymbol{\mu}), \boldsymbol{\mu}\right)=0$ for all $\left|\boldsymbol{\mu}-\boldsymbol{\mu}_{0}\right|<\delta$; i.e., for all $\left|\boldsymbol{\mu}-\boldsymbol{\mu}_{0}\right|<\delta$, the point $\mathbf{x}_{0}^{s}(\boldsymbol{\mu}) \equiv \mathbf{x}^{s}\left(\tau^{s}(\boldsymbol{\mu}), \boldsymbol{\mu}\right) \in l_{0}$. It is similarly shown that there is a unique function $\tau^{u}(\boldsymbol{\mu})$ which is $C^{k}$ or analytic for $\left|\boldsymbol{\mu}-\boldsymbol{\mu}_{0}\right|<\delta$ such that $\tau^{u}(\boldsymbol{\mu}) \rightarrow t_{0}^{u}$ as $\boldsymbol{\mu} \rightarrow \boldsymbol{\mu}_{0}$ and such that $\mathbf{x}_{0}^{u}(\boldsymbol{\mu}) \equiv \mathbf{x}^{u}\left(\tau^{u}(\boldsymbol{\mu}), \boldsymbol{\mu}\right) \in l_{0}$ for all $\left|\boldsymbol{\mu}-\boldsymbol{\mu}_{0}\right|<\delta$.

Definition 1. The displacement function

$$
d(\boldsymbol{\mu}) \equiv\left[\mathbf{x}_{0}^{u}(\boldsymbol{\mu})-\mathbf{x}_{0}^{s}(\boldsymbol{\mu})\right] \wedge \mathbf{u}_{0}
$$

where the points $\mathbf{x}_{0}^{u}(\boldsymbol{\mu}) \in l_{0}$ and $\mathbf{x}_{0}^{s}(\boldsymbol{\mu}) \in l_{0}$ are defined in Lemma 1 for $\mid \boldsymbol{\mu}$ $\boldsymbol{\mu}_{0} \mid<\delta$, the unit vector $\mathbf{u}_{0} \equiv \mathbf{f}\left(\mathbf{x}_{0}, \boldsymbol{\mu}_{0}\right) /\left|\mathbf{f}\left(\mathbf{x}_{0}, \boldsymbol{\mu}_{0}\right)\right|$ and the wedge product of two vectors $\mathbf{x}, \mathbf{y} \in \mathbf{R}^{2}$ is defined by $\mathbf{x} \wedge \mathbf{y}=x_{1} y_{2}-y_{1} x_{2} ;$ cf. Figure 4 . 
By Lemma 1, the displacement function $d(\boldsymbol{\mu})$ is a $C^{k}$ or analytic function for $\left|\boldsymbol{\mu}-\boldsymbol{\mu}_{0}\right|<\delta$ when $\left(1_{\mu}\right)$ is a $C^{k}$ or analytic system respectively. And the system $\left(1_{\mu}\right)$ has a homoclinic loop at the hyperbolic saddle $\mathbf{0}$ iff $d(\boldsymbol{\mu})=0$. The components of the derivative $d_{\mu}\left(\boldsymbol{\mu}_{0}\right)$ are given in the next lemma.

Lemma 2. Under the hypotheses of Theorem 1, for $j=1,2, \ldots, m$,

$$
d_{\mu_{j}}\left(\boldsymbol{\mu}_{0}\right)=-\omega_{0} I_{j} /\left|\mathbf{f}\left(\mathbf{x}_{0}, \boldsymbol{\mu}_{0}\right)\right|
$$

where $\omega_{0}= \pm 1$ according to the orientation of the homoclinic orbit $\gamma_{0}(t), \mathbf{x}_{0}=$ $\gamma_{0}(0)$ and $I_{j}$ is defined by (2).

Before proving Lemma 2, we note that, according to Lemma 1, the separatrices $\Gamma_{\mu}^{s}$ and $\Gamma_{\mu}^{\mu}$ cross the straight line $l_{0}$ at the points $\mathbf{x}_{0}^{s}(\boldsymbol{\mu})$ and $\mathbf{x}_{0}^{u}(\boldsymbol{\mu})$ respectively; cf. Figure 4 . In proving Lemma 2 , it is convenient to define the $C^{k}$ or analytic functions

$$
\mathbf{x}_{\boldsymbol{\mu}}^{s}(t) \equiv \phi\left(t, \mathbf{x}_{0}^{s}(\boldsymbol{\mu}), \boldsymbol{\mu}\right) \quad \text { and } \quad \mathbf{x}_{\mu}^{u}(t) \equiv \phi\left(t, \mathbf{x}_{0}^{u}(\boldsymbol{\mu}), \boldsymbol{\mu}\right)
$$

representing $\Gamma_{\mu}^{s}$ and $\Gamma_{\mu}^{\mu}$ respectively for $t \in \mathbf{R}$ and $\left|\boldsymbol{\mu}-\boldsymbol{\mu}_{0}\right|<\delta$. We only prove Lemma 2 for the case when $j=1$; the other cases follow in exactly the same way.

Proof. According to Definition 1,

$$
d(\boldsymbol{\mu})=\left[\mathbf{x}_{0}^{u}(\boldsymbol{\mu})-\mathbf{x}_{0}^{s}(\boldsymbol{\mu})\right] \wedge \mathbf{u}_{0}
$$

and then, following Duff's notation on p. 22 in [7], we define $n$ to be the coordinate along the normal line $l_{0}$ (with $n>0$ on the exterior of $\Gamma_{0}$ and $n<0$ on the interior of $\Gamma_{0}$ ) and we therefore have

$$
d\left(\boldsymbol{\mu}_{0}\right)=n^{u}\left(0, \boldsymbol{\mu}_{0}\right)-n^{s}\left(0, \boldsymbol{\mu}_{0}\right)
$$

where the functions $n^{u}(t, \boldsymbol{\mu}) \equiv\left[\mathbf{x}_{\mu}^{u}(t)-\mathbf{x}_{0}\right] \wedge \mathbf{u}_{0}$ and $n^{s}(t, \boldsymbol{\mu}) \equiv\left[\mathbf{x}_{\boldsymbol{\mu}}^{s}(t)-\mathbf{x}_{0}\right] \wedge \mathbf{u}_{0}$ are the projections of the vectors $\left[\mathbf{x}_{\mu}^{u}(t)-\mathbf{x}_{0}\right]$ and $\left[\mathbf{x}_{\mu}^{s}(t)-\mathbf{x}_{0}\right]$ onto the line $l_{0}$ respectively. It follows that

$$
d_{\mu_{1}}\left(\boldsymbol{\mu}_{0}\right)=\left[\frac{\partial \mathbf{x}_{0}^{u}}{\partial \mu}\left(\boldsymbol{\mu}_{0}\right)-\frac{\partial \mathbf{x}_{0}^{s}}{\partial \mu}\left(\boldsymbol{\mu}_{0}\right)\right] \wedge \mathbf{u}_{0}=\frac{\partial n^{u}}{\partial \mu_{1}}\left(0, \mu_{0}\right)-\frac{\partial n^{s}}{\partial \mu_{1}}\left(0, \boldsymbol{\mu}_{0}\right) .
$$

But then, as in equation (3.8) in [7],

$$
\frac{\partial n^{s}}{\partial \mu_{1}}(t, \boldsymbol{\mu})=\frac{\omega_{0} \rho(t, \boldsymbol{\mu})}{\left|\mathbf{f}\left(\mathbf{x}_{\boldsymbol{\mu}}^{s}(t), \boldsymbol{\mu}\right)\right|}
$$

where the function $\rho(t, \mu)$ satisfies the first order linear differential equation

$$
\frac{\partial \rho}{\partial t}=\nabla \cdot \mathbf{f}\left(\mathbf{x}_{\boldsymbol{\mu}}^{s}(t), \boldsymbol{\mu}\right) \rho-\mathbf{f} \wedge \mathbf{f}_{\mu_{1}}\left(\mathbf{x}_{\boldsymbol{\mu}}^{s}(t), \boldsymbol{\mu}\right)
$$

obtained from the variational equation of the system $\left(1_{\mu}\right)$ with respect to $\mu_{1}$ along the trajectory $\mathbf{x}_{\mu}^{s}(t)$ of $\left(1_{\mu}\right)$; cf. the derivation of equations (7) and (8) in Appendix II. Note that equation (8) is equivalent to equation (3.14) in [7]. The solution of the linear differential equation (8) is given by

(9) $\left[\rho(t, \boldsymbol{\mu}) e^{-\int_{0}^{t} \nabla \cdot \mathbf{f}\left(\mathbf{x}_{\boldsymbol{\mu}}^{s}\left(t^{\prime}\right), \boldsymbol{\mu}\right) d t^{\prime}}\right]_{t_{0}}^{t_{1}}=-\int_{t_{0}}^{t_{1}} e^{-\int_{0}^{t} \nabla \cdot \mathbf{f}\left(\mathbf{x}_{\boldsymbol{\mu}}^{s}\left(t^{\prime}\right), \boldsymbol{\mu}\right) d t^{\prime}} \mathbf{f} \wedge \mathbf{f}_{\mu_{1}}\left(\mathbf{x}_{\boldsymbol{\mu}}^{s}(t), \boldsymbol{\mu}\right) d t$. 
See equation (3.15) in [7]. Now using the fact that $\mathbf{f}\left(\mathbf{x}_{\boldsymbol{\mu}}^{s}(t), \boldsymbol{\mu}\right) \rightarrow \mathbf{0}$ as $t \rightarrow \infty$, which follows from the fact that $\mathbf{x}_{\mu}^{s}(t)$ approaches the saddle 0 of $\left(1_{\mu}\right)$ as $t \rightarrow \infty$, it follows from equation (7) that $\rho(t, \mu) \rightarrow 0$ as $t \rightarrow \infty$ (since, by Lemma 6 in [17], $\partial \mathbf{x}_{\mu}^{s} / \partial \mu_{1}$ remains bounded as $\left.t \rightarrow \infty\right)$. Thus, setting $t_{0}=0$ and letting $t_{1} \rightarrow \infty$ in (9) yields

$$
\rho(0, \boldsymbol{\mu})=\int_{0}^{\infty} e^{-\int_{0}^{t} \nabla \cdot \mathbf{f}\left(\mathbf{x}_{\mu}^{s}\left(t^{\prime}\right), \boldsymbol{\mu}\right) d t^{\prime}} \mathbf{f} \wedge \mathbf{f}_{\mu_{1}}\left(\mathbf{x}_{\boldsymbol{\mu}}^{s}(t), \boldsymbol{\mu}\right) d t .
$$

Similarly,

$$
\frac{\partial n^{u}}{\partial \mu_{1}}(t, \boldsymbol{\mu})=\frac{\omega_{0} \tilde{\rho}(t, \boldsymbol{\mu})}{\left|\mathbf{f}\left(\mathbf{x}_{\boldsymbol{\mu}}^{u}(t), \boldsymbol{\mu}\right)\right|}
$$

where $\tilde{\rho}(t, \mu)$ satisfies the linear differential equation (8) with $\mathbf{x}_{\mu}^{u}(t)$ in place of $\mathbf{x}_{\boldsymbol{\mu}}^{s}(t)$. It then follows, since $\tilde{\rho}(t, \mu) \rightarrow 0$ as $t \rightarrow-\infty$, by setting $t_{1}=0$ and letting $t_{0} \rightarrow-\infty$ in (9), that

$$
\tilde{\rho}(0, \boldsymbol{\mu})=-\int_{-\infty}^{0} e^{-\int_{0}^{t} \nabla \cdot \mathbf{f}\left(\mathbf{x}_{\boldsymbol{\mu}}^{u}\left(t^{\prime}\right), \boldsymbol{\mu}\right) d t^{\prime}} \mathbf{f} \wedge \mathbf{f}_{\mu_{1}}\left(\mathbf{x}_{\boldsymbol{\mu}}^{u}(t), \boldsymbol{\mu}\right) d t .
$$

But for $\boldsymbol{\mu}=\boldsymbol{\mu}_{0}, \mathbf{x}_{\boldsymbol{\mu}}^{s}(t)=\gamma_{0}(t)=\mathbf{x}_{\boldsymbol{\mu}}^{u}(t)$ for all $t \in \mathbf{R}$ and therefore, it follows from equations (6), (7), (10), (11), and (12) that

$$
\begin{aligned}
d_{\mu_{1}}\left(\boldsymbol{\mu}_{0}\right) & =\frac{\omega_{0}}{\left|\mathbf{f}\left(\mathbf{x}_{0}, \boldsymbol{\mu}_{0}\right)\right|}\left[\tilde{\rho}\left(0, \boldsymbol{\mu}_{0}\right)-\rho\left(0, \boldsymbol{\mu}_{0}\right)\right] \\
& =-\frac{\omega_{0}}{\left|\mathbf{f}\left(\mathbf{x}_{0}, \boldsymbol{\mu}_{0}\right)\right|} \int_{-\infty}^{\infty} e^{-\int_{0}^{t} \nabla \cdot \mathbf{f}\left(\gamma_{0}\left(t^{\prime}\right), \boldsymbol{\mu}_{0}\right) d t^{\prime}} \mathbf{f} \wedge \mathbf{f}_{\mu_{1}}\left(\gamma_{0}(t), \boldsymbol{\mu}\right) d t \\
& =-\frac{\omega_{0} I_{1}}{\left|\mathbf{f}\left(\mathbf{x}_{0}, \boldsymbol{\mu}_{0}\right)\right|}
\end{aligned}
$$

according to the definition of $I_{1}$ in (2). This completes the proof of Lemma 2.

The proof of Theorem 1 is now an easy consequence of the implicit function theorem.

Proof of Theorem 1. Under the hypotheses of Theorem 1, it follows from Lemmas 1 and 2 that for sufficiently small $\left|\boldsymbol{\mu}-\boldsymbol{\mu}_{0}\right|$, the displacement function $d(\mu)$ is a $C^{k}$ or analytic function which satisfies $d\left(\mu_{0}\right)=0$ and $d_{\mu_{1}}\left(\mu_{0}\right)=$ $-\omega_{0} I_{1} /\left|\mathbf{f}\left(\mathbf{x}_{0}, \mu_{0}\right)\right| \neq 0$. It therefore follows from the implicit function theorem for $C^{k}$ or analytic functions, Theorem 10.2.3 or Theorem 10.2.4 on p. 268 in [22] respectively, that there exist a $\delta>0$ and a unique function $h\left(\mu_{2}, \ldots, \mu_{m}\right)$ which satisfies $h\left(\mu_{2}^{(0)}, \ldots, \mu_{m}^{(0)}\right)=\mu_{1}^{(0)}$ and $d\left(h\left(\mu_{2}, \ldots, \mu_{m}\right), \mu_{2}, \ldots, \mu_{m}\right)=$ 0 for $\left|\mu_{j}-\mu_{j}^{(0)}\right|<\delta, j=2, \ldots, m$; furthermore, $h\left(\mu_{2}, \ldots, \mu_{m}\right)$ is a $C^{k}$ or analytic function for $\left|\mu_{j}-\mu_{j}^{(0)}\right|<\delta, j=2, \ldots, m$. It therefore follows from the definition of the displacement function $d(\boldsymbol{\mu})$ that the system $\left(1_{\mu}\right)$ with $\mu_{1}=h\left(\mu_{2}, \ldots, \mu_{m}\right)$ and $\left|\mu_{j}-\mu_{j}^{(0)}\right|<\delta, j=2, \ldots, m$, has a homoclinic orbit $\Gamma_{\mu}$. Furthermore, if $\mu_{1}=h\left(\mu_{2}, \ldots, \mu_{m}\right)$, then $d(\boldsymbol{\mu})=0$, i.e., the functions $\mathbf{x}_{0}^{s}(\boldsymbol{\mu})$ and $\mathbf{x}_{0}^{u}(\boldsymbol{\mu})$ defined in Lemma 1 satisfy $\mathbf{x}_{0}^{s}(\boldsymbol{\mu})=\mathbf{x}_{0}^{u}(\boldsymbol{\mu})$, and by the uniqueness of solutions of $\left(1_{\mu}\right), \mathbf{x}_{\mu}^{s}(t)=\mathbf{x}_{\mu}^{u}(t)$ for all $t \in \mathbf{R}$. It follows that $\Gamma_{\mu}$ is represented by $\mathbf{x}_{\mu}^{s}(t)$ or $\mathbf{x}_{\mu}^{u}(t)$ for $t \in \mathbf{R}$. By continuity of solutions with respect to initial conditions,

$$
\lim _{\boldsymbol{\mu} \rightarrow \boldsymbol{\mu}_{0}} \mathbf{x}_{\boldsymbol{\mu}}^{s}(t)=\lim _{\boldsymbol{\mu} \rightarrow \boldsymbol{\mu}_{0}} \mathbf{x}_{\boldsymbol{\mu}}^{u}(t)=\gamma_{0}(t)
$$


for $t \in \mathbf{R}$, the convergence being uniform on any compact time interval $-a \leq$ $t \leq a$ according to Theorem 4.1 on p. 58 in [23]. And according to the stable manifold theorem for sufficiently large $a>0$, the limits in (13) are uniform for all $t \geq a$ and $t \leq-a$. Thus, given $\varepsilon>0$, there is a $\delta>0$ such that for $t \in \mathbf{R}$ and $\left|\boldsymbol{\mu}-\boldsymbol{\mu}_{0}\right|<\bar{\delta}$ with $\boldsymbol{\mu}=\left(h\left(\mu_{2}, \ldots, \mu_{m}\right), \mu_{2}, \ldots, \mu_{m}\right),\left|\mathbf{x}_{\boldsymbol{\mu}}^{s}(t)-\gamma_{0}(t)\right|<\varepsilon$; i.e., the homoclinic orbit $\Gamma_{\mu}$ is in an $\varepsilon$-neighborhood of $\Gamma_{0}$. Finally, it follows from the uniqueness of the function $h\left(\mu_{2}, \ldots, \mu_{m}\right)$ and the uniqueness of solutions if $\left(1_{\mu}\right)$, i.e., the uniqueness of the separatrices $\Gamma_{\mu}^{u}$ and $\Gamma_{\mu}^{s}$ which approach $\mathbf{0}$ as $t \rightarrow \pm \infty$ respectively, that $\Gamma_{\mu}$ is the only homoclinic orbit in an $\varepsilon$-neighborhood of $\Gamma_{0}$ for $\left|\boldsymbol{\mu}-\mu_{0}\right|<\delta$. This completes the proof of Theorem 1.

Proof of Corollary 1. We shall only prove Corollary 1 in the case when the Poincare map is defined on the interior of $S_{0}$. The other case is treated in a similar fashion. Then under the hypotheses of Theorem 1 , if $\nabla \cdot \mathbf{f}\left(\mathbf{0}, \boldsymbol{\mu}_{0}\right) \neq 0$, it follows from Theorem 44, p. 304 in [1] or from Theorem 1 of $\S 4.7$ of [9] that the separatrix cycle $S_{0}=\Gamma_{0} \cup\{\boldsymbol{0}\}$ is stable or unstable on its interior if $\sigma_{0} \equiv-\operatorname{sgn}\left[\nabla \cdot \mathbf{f}\left(\mathbf{0}, \mu_{0}\right)\right]= \pm 1$ respectively. Thus, there exists an interior neighborhood $N_{\varepsilon}$ of $S_{0}$ such that all trajectories of $\left(1_{\mu_{0}}\right)$ which start in $N_{\varepsilon}$ spiral out toward $S_{0}$ as $\sigma_{0} t \rightarrow \infty$; and, in particular, the trajectory through the point $p_{\varepsilon}$ on $l_{0}$, with coordinate $n=-\varepsilon / 2$, spirals out toward $\Gamma_{0}$ as $\sigma_{0} t \rightarrow \infty$. But then, according to the continuity of solutions with respect to parameters, it follows that there is a $\delta>0$ such that for $\left|\boldsymbol{\mu}-\boldsymbol{\mu}_{0}\right|<\delta$ the trajectory of $\left(1_{\mu}\right)$ through the point $p_{\varepsilon}$ on $l_{0}$ crosses $l_{0}$ again at a point to the right (or left) of $p_{\varepsilon}$ on $l_{0}$ if $\sigma_{0}>0$ (or if $\sigma_{0}<0$ ); cf. Figure 5. Consider the case when $\omega_{0} I_{1}>0$. (The case when $\omega_{0} I_{1}<0$ is treated similarly.) Then according to equation (5) in Lemma $2, d_{\mu_{1}}\left(\mu_{0}\right)<0$. And by the continuity of $d_{\mu_{1}}(\boldsymbol{\mu})$, it follows that there is a $\delta>0$ such that $d_{\mu_{1}}(\boldsymbol{\mu})<0$ for all $\left|\boldsymbol{\mu}-\boldsymbol{\mu}_{0}\right|<$ $\delta$. Let $\mathscr{H}$ be the homoclinic loop bifurcation surface defined in Theorem 1. Then if $\boldsymbol{\mu}^{*} \in \mathscr{H}$ and $\left|\boldsymbol{\mu}^{*}-\boldsymbol{\mu}_{0}\right|<\delta$ we have $d\left(\boldsymbol{\mu}^{*}\right)=0$ and $d_{\mu_{1}}\left(\boldsymbol{\mu}^{*}\right)<$ 0 . It therefore follows from the mean value theorem that for $\left|\boldsymbol{\mu}-\mu_{0}\right|<\delta$, $d(\boldsymbol{\mu})=d_{\mu_{1}}(\tilde{\boldsymbol{\mu}}) \Delta \mu_{1}$ where $\Delta \mu_{1} \equiv \mu_{1}-h\left(\mu_{2}, \ldots, \mu_{m}\right)$ and $\tilde{\boldsymbol{\mu}}=\boldsymbol{\mu}+s \mathbf{e}_{1} \Delta \mu_{1}$ with $\mathbf{e}_{1}=(1,0, \ldots, 0)$ and $0<s<1$. Thus, if $\Delta \mu_{1}>0$ and $\left|\boldsymbol{\mu}-\boldsymbol{\mu}_{0}\right|<\delta$, it follows that $d(\boldsymbol{\mu})<0$; i.e., $n^{u}(0, \boldsymbol{\mu})<n^{s}(0, \boldsymbol{\mu})$ and if $\sigma_{0}>0$, it follows from the Poincaré-Bendixson theorem that $\left(1_{\mu}\right)$ has a stable limit cycle in an $\varepsilon$-neighborhood of $\Gamma_{0}$. Cf. Figure 5(a). Similarly, if $\Delta \mu_{1}<0$ and $\left|\boldsymbol{\mu}-\boldsymbol{\mu}_{0}\right|<\delta$ then $d(\boldsymbol{\mu})>0$; i.e., $n^{u}(0, \boldsymbol{\mu})>n^{s}(0, \boldsymbol{\mu})$ and if $\sigma_{0}<0$, it follows from the Poincaré-Bendixson theorem that $\left(1_{\mu}\right)$ has an unstable limit cycle in an $\varepsilon$-neighborhood of $\Gamma_{0}$. Cf. Figure $5(\mathrm{~b})$. Thus, for $\left|\boldsymbol{\mu}-\boldsymbol{\mu}_{0}\right|<\delta,\left(1_{\mu}\right)$ has a limit cycle $\Gamma_{\mu}$ in an $\varepsilon$-neighborhood of $\Gamma_{0}$ if $\omega_{0} I_{1} \sigma_{0} \Delta \mu_{1}>0$. The uniqueness of the limit cycle follows from the fact that if $\nabla \cdot \mathbf{f}(\mathbf{0}, \boldsymbol{\mu}) \neq 0$ then at most one limit cycle bifurcates from $\Gamma_{0}$ as $\mu$ varies from $\mu_{0} ; \mathrm{cf}$. Theorem $47, \mathrm{p}$. 312 in [1] or Theorem 2 in $\$ 4.7$ of [9]. And the hyperbolicity of the limit cycle $\Gamma_{\mu}$ follows from Theorems 42 and 47 in [1]. This completes the proof of part (a) of Corollary 1. Part (b) of Corollary 1 follows immediately from Theorem 1 and its proof. And part (c) of Corollary 1 follows from the fact that if $\nabla \cdot \mathbf{f}\left(\mathbf{0}, \boldsymbol{\mu}_{0}\right) \neq 0$ then $\left(1_{\mu}\right)$ has at most one limit cycle in an $\varepsilon$-neighborhood of $\Gamma_{0}$ (according to Theorem 47, p. 312 in [1]) and an argument similar to that used above (based on the Poincaré-Bendixson theorem) which implies that 


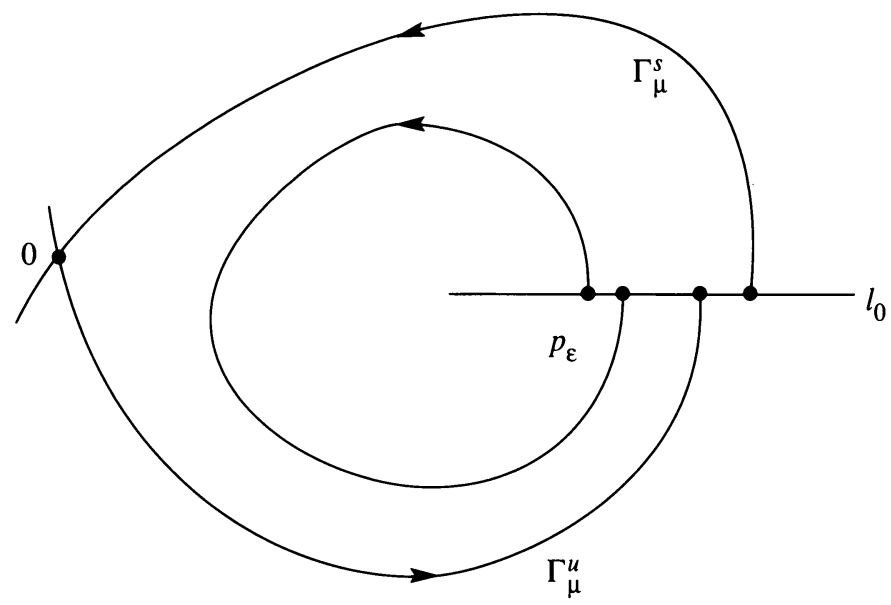

(a) $\sigma_{0}>0$

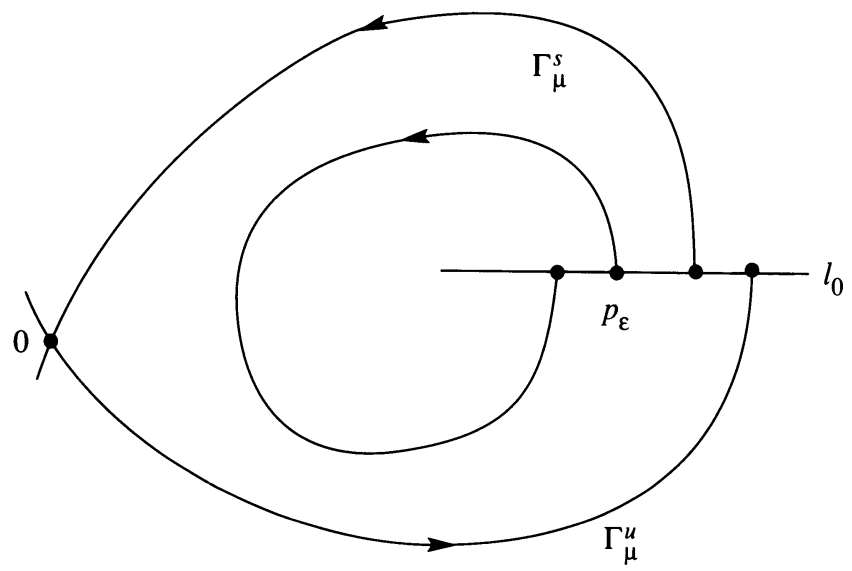

(b) $\sigma_{0}<0$

FIGURE 5. For $\omega_{0}>0$ and $I_{1}>0$, the system $\left(1_{\mu}\right)$ has a limit cycle if $\sigma_{0} d(\boldsymbol{\mu})<0$, i.e., if $\sigma_{0} \Delta \mu_{1}>0$

if $\left(1_{\mu}\right)$ has a unique limit cycle in an $\varepsilon$-neighborhood of $\Gamma_{0}$, then it must be a semistable limit cycle. But then, according to the theory of rotated vector fields in [7] or [17], an arbitrarily small rotation of $\left(1_{\mu}\right)$ would cause the semistable limit cycle to split into two limit cycles contradicting the uniqueness result in Theorem 47 on p. 312 in [1]. Thus, for $\omega_{0} I_{1} \sigma_{0} \Delta \mu_{1}<0,\left(1_{\mu}\right)$ has no limit cycle in an $\varepsilon$-neighborhood of $\Gamma_{0}$ and by part (b), $\left(1_{\mu}\right)$ has no separatrix cycle in an $\varepsilon$-neighborhood of $\Gamma_{0}$ for $\Delta \mu_{1} \neq 0$. This completes the proof of Corollary 1 .

Proof of Corollary 2. We only prove Corollary 2 in the case when the Poincare map for $\left(1_{\mu}\right)$ is defined on an interior neighborhood of $S_{0}$. The other case is proved in a similar fashion. It then follows from the continuity of solutions of $\left(1_{\mu}\right)$ with respect to initial conditions and parameters, Lemma 5 on p. 293 in [1] and equation (5) on p. 301 in [1] that the separatrix cycle $S_{0}=\Gamma_{0} \cup\{0\}$ of $\left(1_{\mu_{0}}\right)$ is stable or unstable on its interior according to whether the integral $I_{0}$ 
along $\gamma_{0}(t)$, defined in (4), is positive or negative respectively. A repeat of the argument used in the proof of Corollary 1 (based on the Poincaré-Bendixson theorem) can then be used to show that if $\omega_{0} I_{0} I_{1} \Delta \mu_{1}>0$ then for $\left|\boldsymbol{\mu}-\boldsymbol{\mu}_{0}\right|<\delta$, $\left(1_{\mu}\right)$ has at least one limit cycle $\Gamma_{\mu}$ in an $\varepsilon$-neighborhood of $\Gamma_{0}$ (cf. Figure 5). But according to Roussarie's theorem in [37], under the hypotheses of Corollary 2 , $\left(1_{\mu}\right)$ has at most two limit cycles in an $\varepsilon$-neighborhood of $\Gamma_{0}$. Therefore, $\Gamma_{\mu}$ is a hyperbolic limit cycle and it is unique. This completes the proof of part (a) of Corollary 2. Part (b) follows immediately from Theorem 1 and its proof. And part (c) follows as in the last part of the proof of Corollary 1 using Roussarie's theorem in [37]. This completes the proof of Corollary 2.

Note that under the hypotheses of Corollary 2, it follows from Lemma 6 on p. 302 in [1] that $\nabla \cdot \mathbf{f}\left(\mathbf{0}, \boldsymbol{\mu}_{0}\right)=0$ and we cannot use Theorem 47 on p. 312 in [1] to establish that there is only one limit cycle near $\Gamma_{0}$ if $\omega_{0} I_{0} I_{1} \Delta \mu_{1}>0$ and no limit cycle near $\Gamma_{0}$ if $\omega_{0} I_{0} I_{1} \Delta \mu_{1}<0$. In fact, an example, showing that all three of the possibilities listed in part (c) of Corollary 2 can occur when $\omega_{0} I_{0} I_{1} \Delta \mu_{1}<0$, is given at the end of the next section.

\section{MUltiple Limit CYCLE BIFURCATION SURFACES}

Suppose that the $C^{k}$ or analytic system $\left(1_{\mu}\right)$, with $2 \leq k \leq \infty, \mathbf{x} \in \mathbf{R}^{2}$, $\boldsymbol{\mu} \in \mathbf{R}^{m}$, and $m \geq 2$, has a multiplicity-two limit cycle

$$
L_{0}: \mathbf{x}=\mathbf{x}_{0}(t), \quad 0 \leq t \leq T_{0},
$$

of period $T_{0}$ for $\boldsymbol{\mu}=\boldsymbol{\mu}_{0}$. Then typically as $\boldsymbol{\mu}$ varies from $\boldsymbol{\mu}_{0}$ in the parameter space $\mathbf{R}^{m}, L_{0}$ either vanishes or it splits into two simple limit cycles. However, if one of the integrals

$$
J_{j} \equiv \int_{0}^{T_{0}} e^{\left.-\int_{0}^{t} \nabla \cdot \mathbf{f}\left(\mathbf{x}_{0}(s), \mu_{0}\right), \mu_{0}\right) d s} \mathbf{f} \wedge \mathbf{f}_{\mu_{j}}\left(\mathbf{x}_{0}(t), \boldsymbol{\mu}_{0}\right) d t
$$

for $j=1, \ldots, m$, does not vanish, then locally there is an $(m-1)$ dimensional, $C^{k}$ or analytic manifold $\mathscr{C}$ on which $\left(1_{\mu}\right)$ has a multiplicity-two limit cycle. This is made precise in the following theorem where we assume that $J_{1} \neq 0$, let $\omega_{0}= \pm 1$ according to whether the limit cycle $L_{0}$ is positively or negatively oriented respectively, and let $\sigma_{0}= \pm 1$ according to whether $L_{0}$ is stable or unstable on its interior respectively. Note that it then follows that $\sigma_{0}= \pm 1$ according to whether $L_{0}$ is unstable or stable on its exterior respectively.

Theorem 2. Suppose that for $\boldsymbol{\mu}=\mu_{0}$ the $C^{k}$ or analytic system $\left(1_{\mu}\right)$ with $2 \leq k \leq \infty$ and $m \geq 2$ has a multiplicity-two limit cycle $\mathbf{x}_{0}(t)$ of period $T_{0}$ and that $J_{1} \neq 0$. Then given $\varepsilon>0$, there exists $a \delta>0$ and a unique function $g\left(\mu_{2}, \ldots, \mu_{m}\right)$ with $g\left(\mu_{2}^{(0)}, \ldots, \mu_{m}^{(0)}\right)=\mu_{1}^{(0)}$, defined and $C^{k}$ or analytic for $\left|\mu_{j}-\mu_{j}^{(0)}\right|<\delta, j=2, \ldots, m$, such that the system $\left(1_{\mu}\right)$ with $\mu_{1}=g\left(\mu_{2}, \ldots, \mu_{m}\right)$ and $\left|\mu_{j}-\mu_{j}^{(0)}\right|<\delta$ for $j=2, \ldots, m$ has a unique semistable limit cycle of multiplicity two, $L_{\mu}: \mathbf{x}=\mathbf{x}_{\mu}(t)$ of period $T_{\mu}$ in an $\varepsilon$-neighborhood of $L_{0}$ where $T_{\mu} \rightarrow T_{0}$ as $\mu \rightarrow \mu_{0}$; i.e., $\left(1_{\mu}\right)$ has a unique, local, $(m-1)$-dimensional saddle-node bifurcation surface $\mathscr{C}: \mu_{1}=g\left(\mu_{2}, \ldots, \mu_{m}\right)$ of multiplicity-two limit cycles of $\left(1_{\mu}\right)$ in the sense that for $\mu \in \mathscr{C}$, the system $\left(1_{\mu}\right)$ has a saddle-node bifurcation at each multiplicity-two limit cycle $L_{\mu}$ 


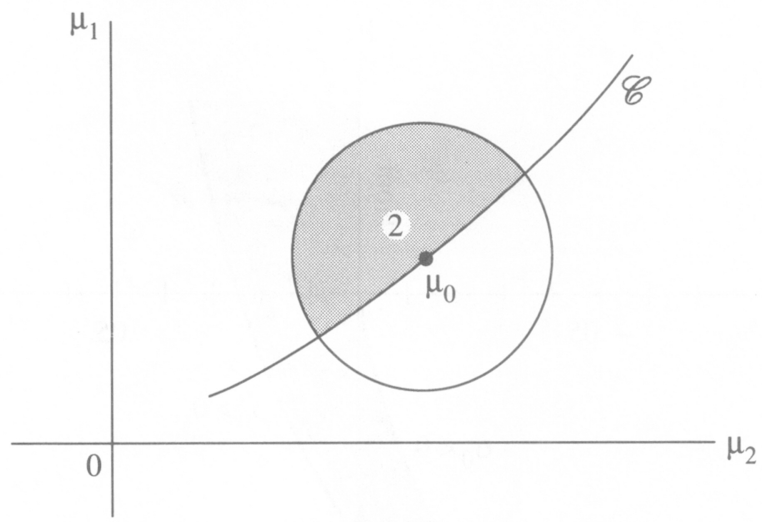

FiguRE 6. If $\omega_{0} \sigma_{0} J_{1}>0$, then locally $\left(1_{\mu}\right)$ has two simple limit cycles near $L_{0}$ if $\mu_{1}>g\left(\mu_{2}, \ldots, \mu_{m}\right)$ and no limit cycle near $L_{0}$ if $\mu_{1}<g\left(\mu_{2}, \ldots, \mu_{m}\right)$

of $\left(1_{\boldsymbol{\mu}}\right)$ with $\boldsymbol{\mu} \in \mathscr{C}$ where $L_{\boldsymbol{\mu}}$ splits into a simple stable and a simple unstable limit cycle if $\omega_{0} \sigma_{0} J_{1} \Delta \mu_{1}>0$ and $L_{\mu}$ vanishes if $\omega_{0} \sigma_{0} J_{1} \Delta \mu_{1}<0$ where $\Delta \mu_{1} \equiv \mu_{1}-g\left(\mu_{2}, \ldots, \mu_{m}\right)$. Cf. Figure 6 .

Remark 3. For $m=2$, Theorem 2 reduces to Theorem 3 in [4]. Also, in the terminology of [24], the manifold $\mathscr{C}$ is a local saddle-node bifurcation surface of multiplicity-two limit cycles of $\left(1_{\mu}\right)$ and the set of $C^{k}$ or analytic vector fields $\mathbf{f}(\mathbf{x}, \boldsymbol{\mu})$, with $\mathbf{x} \in \mathbf{R}^{2}$ and $\boldsymbol{\mu} \in \mathbf{R}^{m}$, with $m \geq 2$, which satisfy the hypotheses of Theorem 2, is an open dense subset of the set of all $C^{k}$ or analytic vector fields $\mathbf{f}(\mathbf{x}, \boldsymbol{\mu})$ having a multiplicity-two limit cycle $L_{0}$ at $\boldsymbol{\mu}=\boldsymbol{\mu}_{0}$. Furthermore, for $k \geq 2$, multiplicity- $k$ limit cycle bifurcation surfaces, their existence, extension and termination, are studied in detail in [24].

Remark 4. If the system $\left(1_{\mu}\right)$ defines a one-parameter family of rotated vector fields with parameter $\mu_{1}$ then $J_{1}>0$, cf. [7] or [17], and Theorem 2 applies; furthermore, in the case when $\left(1_{\mu}\right)$ is an analytic system, the rotated vector field theory in [7] and [17] and the uniqueness of analytic continuations in [18] can be used to establish the existence of a global, analytic, saddle-node bifurcation surface of multiplicity-two limit cycles, $\mathscr{C}$.

An excellent example illustrating the theory in this paper is the quadratic system

$$
\begin{aligned}
& \dot{x}=\left(\mu_{2}-\mu_{1}-1\right) x+y-x^{2}+\left(1+\mu_{1}\right) x y-y^{2}, \\
& \dot{y}=2 x+2 x^{2} / 3-x y
\end{aligned}
$$

studied by Rousseau in [12]. Rousseau established the existence of global homoclinic loop and saddle-node bifurcation surfaces $\mathscr{H}$ and $\mathscr{C}$ for this system in [12] along with the fact that there is a supercritical (or subcritical) Hopf bifurcation at $\mu_{2}=0$ if $\mu_{1}>0$ (or if $\mu_{1}<0$ ). Cf. Figure 7. The shape of the bifurcation curves $\mathscr{C}$ and $\mathscr{H}$ shown in Figure 7 was determined numerically. Also, it was determined numerically by the author that the intersection of $\mathscr{H}$ and $\mathscr{C}$ occurs at $\boldsymbol{\mu}_{0}^{*} \cong(-1.065,-.065)$, and that if $\boldsymbol{\mu}=\boldsymbol{\mu}_{0}^{*}$, then $\nabla \cdot \mathbf{f}\left(\mathbf{0}, \boldsymbol{\mu}_{0}^{*}\right)=0, I_{0} \cong-.52, I_{1} \cong .59$, and $I_{2} \cong-4.2$. It follows from the above differential equations that $\omega_{0}=+1$ and that $\nabla \cdot \mathbf{f}\left(\mathbf{0}, \boldsymbol{\mu}_{0}\right) \neq 0$ if $\boldsymbol{\mu}_{0} \neq \boldsymbol{\mu}_{0}^{*}$; 


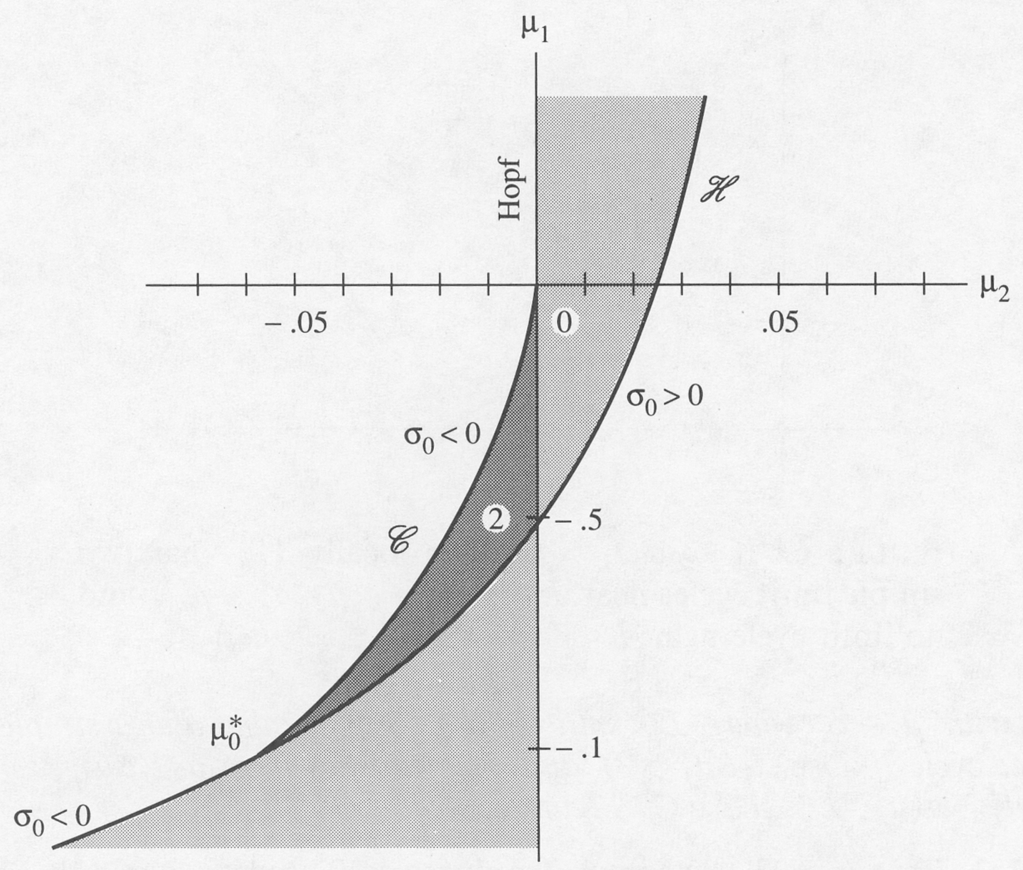

FIGURE 7. The bifurcation diagram showing the homoclinic loop and multiplicity-two bifurcation curves $\mathscr{H}$ and $\mathscr{C}$ for Rousseau's quadratic system in [12]

in fact $\sigma_{0}=-\operatorname{sgn}\left[\nabla \cdot \mathbf{f}\left(\mathbf{0}, \boldsymbol{\mu}_{0}\right)\right]$ is positive (or negative) if $\mu_{1}^{(0)}>\mu_{1}^{(0) *}$ (or if $\mu_{1}^{(0)}<\mu_{1}^{(0) *}$ ) as shown in Figure 7. The side of the homoclinic loop bifurcation surface $\mathscr{H}$ on which a limit cycle bifurcates from the homoclinic loop $\Gamma_{0}$ is therefore determined by Corollary 1 for $\mu_{0} \neq \mu_{0}^{*}$ and by Corollary 2 for $\boldsymbol{\mu}_{0}=\boldsymbol{\mu}_{0}^{*}$. The results are consistent with Rousseau's results shown in Figure 7. It was shown by the author in [25] that as $\mu_{0} \rightarrow \mu_{0}^{*}$ along the curve $\mathscr{C}$, the quantity $J_{1}$, defined by (14), approaches the quantity $\frac{1}{2} I_{1}$, defined by (2), with $\boldsymbol{\mu}_{0}=\boldsymbol{\mu}_{0}^{*}$ in (2); cf. Lemma 4 in $\S 4$. Thus, since $I_{1} \cong .59>0$ for $\boldsymbol{\mu}_{0}=\boldsymbol{\mu}_{0}^{*}$, it follows that $J_{1}>0$, at least for $\mu_{0}$ near $\mu_{0}^{*}$ on $\mathscr{C}$. (This is also borne out by numerical computation.) And since the semistable, multiplicity-two limit cycle $L_{0}$ for this system, which occurs when $\boldsymbol{\mu} \in \mathscr{C}$, is stable on its exterior (which follows since the critical point inside $L_{0}$ is stable), we have $\sigma_{0}=-1$ along $\mathscr{C}$. Thus, according to the result in Theorem 2, two limit cycles bifurcate from $L_{0}$ for $\Delta \mu_{1}<0$, i.e., for $\mu_{1}<g\left(\mu_{2}\right)$, since $\omega_{0} \sigma_{0} J_{1}<0$; and once again this is consistent with Rousseau's results shown in Figure 7. The phase portraits for this quadratic system are given by Rousseau in [12] and in Figure 9 of this paper. The new result that the bifurcation curves $\mathscr{H}$ and $\mathscr{C}$ in Figure 7 intersect at a flat contact at $\boldsymbol{\mu}=\boldsymbol{\mu}_{0}^{*}$ follows from Corollary 4 in $\S 4$.

It should be noted that in any small neighborhood of the point $\mu_{0}^{*}$ in Figure 7 there is a (unique) limit cycle of Rousseau's system for $\mu_{1}<h\left(\mu_{2}\right)$ and no unique stable or unstable limit cycle for $\mu_{1}>h\left(\mu_{2}\right)$; in fact, there are either two simple limit cycles; one multiplicity-two limit cycle, or no limit cycles for $\mu_{1}>$ $h\left(\mu_{2}\right)$ and $\boldsymbol{\mu}$ in a small neighborhood of $\boldsymbol{\mu}_{0}^{*}$. This is consistent with the results 
in Corollary 2 (since $\omega_{0} I_{0} I_{1}<0$ in this example). Other interesting examples with homoclinic loop and multiplicity-two limit cycle bifurcation surfaces can be found in [11-15].

The proof of Theorem 2 follows directly from the Weierstrass preparation theorem (for analytic functions) and the Malgrange preparation theorem (for $C^{k}$ functions) in view of the following classical result for the derivatives $d_{\mu_{j}}\left(0, \mu_{0}\right)$ of the displacement function $d(n, \mu)$ which is defined and $C^{k}$ or analytic in a neighborhood of the point $\left(0, \mu_{0}\right)$ according to Lemma 1.1 in [28]. Note that as in [28] the displacement function along the transversal $i_{0}$, $d(n, \boldsymbol{\mu}) \equiv h(n, \boldsymbol{\mu})-n$ where $h(n, \boldsymbol{\mu})$ is the Poincare map for the system $\left(1_{\boldsymbol{\mu}}\right)$ along $l_{0}$ and $n$ is the coordinate along $l_{0}$ as in $\S 1$.

Lemma 3. Under the hypotheses of Theorem 2 , for $j=1,2, \ldots, m$,

$$
d_{\mu_{j}}\left(0, \mu_{0}\right)=-\omega_{0} J_{j} /\left|\mathbf{f}\left(\mathbf{x}_{0}, \boldsymbol{\mu}_{0}\right)\right|
$$

where $\omega_{0}= \pm 1$ according to the orientation of the multiplicity-two limit cycle $\mathbf{x}_{0}(t), \mathbf{x}_{0}=\mathbf{x}_{0}(0)$, and $J_{j}$ is defined by (14).

This classical formula is equation (36), p. 384 in [1] with $\int_{0}^{T_{0}} \nabla \cdot \mathbf{f}\left(\mathbf{x}_{0}(t), \mu_{0}\right) d t$ $=0$ since $\mathbf{x}_{0}(t)$ is a multiple limit cycle; cf. Lemma 2 in [4] and equation (3.17) in [7]. As was pointed out by Chicone and Jacobs [2], equation (15) is one of the most useful results in the study of bifurcations of limit cycles of planar dynamical systems.

Proof of Theorem 2. Under the hypotheses of Theorem 2, it follows from Lemma 3 and the fact that $L_{0}$ is a multiplicity-two limit cycle of $\left(1_{\mu_{0}}\right)$, cf. Definition 2 on p. 198 in [9], that $d\left(0, \mu_{0}\right)=d_{n}\left(0, \mu_{0}\right)=0, d_{n n}\left(0, \mu_{0}\right) \neq 0$, and $d_{\mu_{1}}\left(0, \mu_{0}\right) \neq 0$, where $n$ is the coordinate along the normal line $l_{0}$ to the limit cycle $L_{0}$ at the point $\mathbf{x}_{0}$. Furthermore, there is a $\delta>0$ such that $d(n, \mu)$ is a $C^{k}$ or analytic function of $n$ and $\boldsymbol{\mu}$ for $|n|<\delta$ and $\left|\boldsymbol{\mu}-\boldsymbol{\mu}_{0}\right|<\delta$; cf. Lemma 1.1 in [28] or Lemma 13 and Remark 1 on p. 74 in [29]. Thus, according to the Weierstrass preparation theorem for analytic functions or the Malgrange preparation theorem for $C^{k}$ functions, cf. [1 and 27], there exists a $\delta>0$ such that the displacement function

$$
d(n, \boldsymbol{\mu})=\left[n^{2}+A_{1}(\boldsymbol{\mu}) n+A_{2}(\boldsymbol{\mu})\right] \Phi(n, \boldsymbol{\mu})
$$

where $A_{1}(\mu), A_{2}(\mu)$, and $\Phi(n, \mu)$ are $C^{k}$ or analytic functions for $|n|<\delta$ and $\left|\boldsymbol{\mu}-\mu_{0}\right|<\delta ; A_{1}\left(\mu_{0}\right)=A_{2}\left(\mu_{0}\right)=0, \Phi\left(0, \mu_{0}\right) \neq 0$; and $\partial A_{2}\left(\mu_{0}\right) / \partial \mu_{1} \neq 0$ since $d_{\mu_{1}}\left(0, \mu_{0}\right) \neq 0$. It then follows from (16) that

$$
d_{n}(n, \boldsymbol{\mu})=\left[2 n+A_{1}(\boldsymbol{\mu})\right] \Phi(n, \boldsymbol{\mu})+\left[n^{2}+A_{1}(\boldsymbol{\mu}) n+A_{2}(\boldsymbol{\mu})\right] \Phi_{n}(n, \boldsymbol{\mu})
$$

and

$$
\begin{aligned}
d_{n n}(\mu, \boldsymbol{\mu})= & 2 \Phi(n, \boldsymbol{\mu})+2\left[2 n+A_{1}(\boldsymbol{\mu})\right] \Phi_{n}(n, \boldsymbol{\mu}) \\
& +\left[n^{2}+A_{2}(\boldsymbol{\mu}) n+A_{2}(\boldsymbol{\mu})\right] \Phi_{n n}(n, \boldsymbol{\mu})
\end{aligned}
$$

for $|n|<\delta$ and $\left|\boldsymbol{\mu}-\mu_{0}\right|<\delta$. And from equations (16), (17), and (18) we see that if $2 n+A_{1}(\boldsymbol{\mu})=0$ and $n^{2}+A_{1}(\boldsymbol{\mu}) n+A_{2}(\boldsymbol{\mu})=0$, then $\left(1_{\mu}\right)$ has a multiplicity-two limit cycle through the point on $l_{0}$ with coordinate $n$. So we set $n=-A_{1}(\boldsymbol{\mu}) / 2$ and find that, from (16), $d\left(-A_{1}(\boldsymbol{\mu}) / 2, \boldsymbol{\mu}\right)=0$ iff the $C^{k}$ or analytic function

$$
G(\boldsymbol{\mu}) \equiv \frac{1}{4} A_{1}^{2}(\boldsymbol{\mu})-A_{2}(\boldsymbol{\mu})=0
$$


(since by continuity $\Phi\left(n, \mu_{0}\right) \neq 0$ for $|n|<\delta$ and $\left.\left|\boldsymbol{\mu}-\boldsymbol{\mu}_{0}\right|<\delta\right)$. But $G\left(\boldsymbol{\mu}_{0}\right)=$ 0 since $A_{1}\left(\mu_{0}\right)=A_{2}\left(\mu_{0}\right)=0$ and

$$
\frac{\partial G}{\partial \mu_{1}}\left(\boldsymbol{\mu}_{0}\right)=-\frac{\partial A_{2}}{\partial \mu_{1}}\left(\boldsymbol{\mu}_{0}\right) \neq 0
$$

since $d_{\mu_{1}}\left(0, \mu_{0}\right) \neq 0$. Thus, it follows from the implicit function theorem for $C^{k}$ or analytic functions, Theorem 10.2 .3 or Theorem 10.2 .4 on p. 268 in [22] respectively, that there exists a $\delta>0$ and a unique function $g\left(\mu_{2}, \ldots, \mu_{m}\right)$ such that $g\left(\mu_{2}^{(0)}, \ldots, \mu_{m}^{(0)}\right)=\mu_{1}^{(0)}$ and $G\left(g\left(\mu_{2}, \ldots, \mu_{m}\right), \mu_{2}, \ldots, \mu_{m}\right)=0$ for $\left|\mu_{j}-\mu_{j}^{(0)}\right|<\delta, j=2, \ldots, m$; furthermore, $g\left(\mu_{2}, \ldots, \mu_{m}\right)$ is $C^{k}$ or analytic for $\left|\mu_{j}-\mu_{j}^{(0)}\right|<\delta, j=2, \ldots, m$. We have therefore shown that if the point $\boldsymbol{\mu} \in \mathbf{R}^{m}$ with $\left|\boldsymbol{\mu}-\boldsymbol{\mu}_{0}\right|<\delta$ lies on the $C^{k}$ or analytic surface $\mathscr{C}: \mu_{1}=g\left(\mu_{2}, \ldots, \mu_{m}\right)$ and if

$$
n=-\frac{1}{2} A_{1}(\boldsymbol{\mu})
$$

then, from $(16)-(18)$, it follows that $d(n, \boldsymbol{\mu})=d_{n}(n, \boldsymbol{\mu})=0$ and $d_{n n}(n, \boldsymbol{\mu})=$ $2 \Phi(n, \boldsymbol{\mu}) \neq 0$; i.e., $\left(1_{\mu}\right)$ with $\boldsymbol{\mu} \in \mathscr{C}$ has a multiplicity-two, semistable limit cycle $L_{\mu}$ through the point on $l_{0}$ with coordinate $n(\boldsymbol{\mu})$ determined by (19). Furthermore, on the side of the surface $\mathscr{C}$ where $G(\boldsymbol{\mu})>0$, the equation $d(n(\boldsymbol{\mu}), \boldsymbol{\mu})=0$ with $n(\boldsymbol{\mu})$ given by (19) has two real solutions given by

$$
n^{ \pm}(\boldsymbol{\mu})=-\frac{1}{2} A_{1}(\boldsymbol{\mu}) \pm \sqrt{A_{1}^{2}(\boldsymbol{\mu}) / 4-A_{2}(\boldsymbol{\mu})}
$$

and on the side of $\mathscr{C}$ where $G(\boldsymbol{\mu})<0, d(n(\boldsymbol{\mu}), \boldsymbol{\mu})=0$ has no real solution; i.e., locally, $\left(1_{\mu}\right)$ has two limit cycles if $G(\boldsymbol{\mu})>0$ and no limit cycle if $G(\boldsymbol{\mu})<0$. And it follows from (17) that if $n^{ \pm}(\boldsymbol{\mu})$ is given by (20) then $d_{n}\left(n^{ \pm}(\boldsymbol{\mu}), \boldsymbol{\mu}\right) \gtrless 0$ respectively; i.e., locally, $\left(1_{\mu}\right)$ has two simple (unstable or stable) limit cycles if $G(\boldsymbol{\mu})>0$. The fact that the side of $\mathscr{C}$ on which $\left(1_{\mu}\right)$ has two simple limit cycles is determined by the sign of $\omega_{0} \sigma_{0} J_{1} \Delta \mu_{1}$, with $\Delta \mu_{1}=\mu_{1}-g\left(\mu_{2}, \ldots, \mu_{m}\right)$, follows exactly as in Theorem 3 in [4] or as in the theorem in the appendix of [28]; and the fact that the period $T_{\mu}$ of the limit cycle $L_{\mu}$ of $\left(1_{\mu}\right)$ with $\boldsymbol{\mu} \in \mathscr{C}$ approaches $T_{0}$ as $\boldsymbol{\mu}$ approaches $\boldsymbol{\mu}_{0}$ follows exactly as in the proof of the existence of the Poincaré map, cf., e.g., Theorem 1 on p. 194 in [9]. This completes the proof of Theorem 2 .

Remark 5. The surface

$$
\tilde{\mathscr{C}}: n=-\frac{1}{2} A_{1}\left(g\left(\mu_{2}, \ldots, \mu_{m}\right), \mu_{2}, \ldots, \mu_{m}\right)
$$

defined in the proof of Theorem 2 is the lifting of the surface $\mathscr{C}$ from the parameter space $\mathbf{R}^{m}$ into the $(\boldsymbol{\mu}, n)$ space $\mathbf{R}^{m+1}$; locally it represents the bifurcation surface $d(n, \boldsymbol{\mu})=0$ as defined in [26].

Remark 6. In [24] it is shown that for an analytic system $\left(1_{\mu}\right)$, any local oneparameter family of multiplicity-two limit cycles $\mathscr{C}$ (such as that established in Theorem 2 with $m=2$ ) can be continued to a unique, maximal, one-parameter family of multiplicity-two limit cycles which is either unbounded or cyclic or which terminates at a degenerate Hopf bifurcation of order $k \geq 2$ (which is generically of order $k=2$ ) or at a degenerate homoclinic loop bifurcation of order $k \geq 2$ (which is generically of order $k=2$ ) or at a degenerate critical point of $\left(1_{\mu}\right)$. It is also shown in [24] that this same type of termination 
principle holds for one-parameter families of multiplicity- $k$ limit cycles $\mathscr{C}_{k}$ (whose local existence is established in [24]). That work extends the author's planar termination principle for one-parameter families of simple limit cycles in [28] to one-parameter families of multiple limit cycles.

In the next section we show that if a one-parameter family of multiplicity-two limit cycles $\mathscr{C}$ of $\left(1_{\mu}\right)$ terminates at a homoclinic loop bifurcation at $\boldsymbol{\mu}=\boldsymbol{\mu}_{0}$, then generically there is a unique homoclinic loop bifurcation curve $\mathscr{H}$ through the point $\boldsymbol{\mu}_{0} \in \mathbf{R}^{2}$ and the curves $\mathscr{C}$ and $\mathscr{H}$ have a flat contact at $\boldsymbol{\mu}=\boldsymbol{\mu}_{0}$.

\section{INTERSECTIONS OF $\mathscr{C}$ AND $\mathscr{H}$}

The discussion in this section is restricted to the case where $\boldsymbol{\mu} \in \mathbf{R}^{2}$. We therefore let $\boldsymbol{\mu}=(\alpha, \beta)$ and begin with a few preliminary definitions.

Definition 2. If $\kappa \in C(0, \infty)$ and there are positive constants $\delta, k_{0}$, and $a_{0}>$ 1 such that for $0<\beta<\delta\left|\kappa(\beta) a_{0}^{1 / \beta}\right| \leq k_{0}$, then $\kappa(\beta)$ is exponentially flat at $\beta=0$.

Definition 3. If $\kappa \in C^{\infty}(0, \infty)$ and $\kappa\left(0^{+}\right)=\kappa^{\prime}\left(0^{+}\right)=\cdots=0$, then $\kappa(\beta)$ has a flat contact (or a contact of infinite order) with the $\beta$-axis at $\beta=0$; and if $g, h \in C^{\infty}\left(\beta_{0}, \infty\right)$ and $g^{(k)}\left(\beta_{0}^{+}\right)=h^{(k)}\left(\beta_{0}^{+}\right)$for $k=0,1,2, \ldots$, then $g$ and $h$ have a flat contact at the point $\left(\alpha_{0}, \beta_{0}\right) \in \mathbf{R}$ where $\alpha_{0}=g\left(\beta_{0}^{+}\right)=h\left(\beta_{0}^{+}\right)$.

If $\kappa \in C^{\infty}(0, \infty)$ and $\kappa\left(0^{+}\right)$exists then we can define the right-hand derivative at $\beta=0$,

$$
\kappa_{+}^{\prime}(0)=\lim _{\beta \rightarrow 0+} \frac{\kappa(\beta)-\kappa\left(0^{+}\right)}{\beta}
$$

if the limit exists. If $\kappa_{+}^{\prime}(0)$ and $\kappa^{\prime}\left(0^{+}\right)$both exist then it follows from the mean value theorem that $\kappa_{+}^{\prime}(0)=\kappa^{\prime}\left(0^{+}\right)$. Furthermore, it is not difficult to show that if $\kappa \in C^{\infty}(0, \infty)$ is exponentially flat at $\beta=0$ and if $\kappa\left(0^{+}\right), \kappa^{\prime}\left(0^{+}\right), \ldots$ exist, then $\kappa\left(0^{+}\right)=\kappa^{\prime}\left(0^{+}\right)=\cdots=0$, i.e., $\kappa(\beta)$ has a flat contact with the $\beta$-axis at $\beta=0$; however, $\kappa(\beta)$ being exponentially flat at $\beta=0$ does not imply that $\kappa(\beta)$ has a flat contact with the $\beta$-axis at $\beta=0$ unless we know that $\kappa^{\prime}\left(0^{+}\right)$, $\kappa^{\prime \prime}\left(0^{+}\right), \ldots$ all exist. For example, the function

$$
\kappa(\beta)=\mathbf{e}^{-1 / \beta} \sin \left(\mathbf{e}^{1 / \beta}\right) \in C^{\infty}(0, \infty)
$$

is exponentially flat at $\beta=0, \kappa\left(0^{+}\right)=0$, and $\kappa_{+}^{\prime}(0)=0$, but $\kappa^{\prime}\left(0^{+}\right)$does not exist and $\kappa(\beta)$ does not have a flat contact with the $\beta$-axis at $\beta=0$. Thus, in the context of Definitions 2 and 3, Dumortier, Roussarie and Sotomayor [15] only show that the surfaces $\mathscr{C}$ and $\mathscr{H}$, defined in [15], have an exponentially flat intersection, but they do not show that $\mathscr{C}$ and $\mathscr{H}$ have a flat contact since, as they point out in their final remark, the derivatives $\Gamma^{\prime}\left(0^{+}\right), \Gamma^{\prime \prime}\left(0^{+}\right), \ldots$ may not exist. However, according to Theorem 5 or its corollary in this paper, the surfaces $\mathscr{C}$ and $\mathscr{H}$ in [15] do, in fact, intersect at a flat contact and $\Gamma^{\prime}\left(0^{+}\right)=$ $\Gamma^{\prime \prime}\left(0^{+}\right)=\cdots=0$.

In this section, we show that if a $C^{\infty}$ system $\left(1_{\mu}\right)$ with $\boldsymbol{\mu} \in \mathbf{R}^{2}$ has a multiplicity-two limit cycle bifurcation curve $\mathscr{C}$ which intersects a homoclinic loop bifurcation curve $\mathscr{H}$ at a point $\boldsymbol{\mu}_{0} \in \mathbf{R}^{2}$, then typically $\mathscr{C}$ and $\mathscr{H}$ have a flat contact at the point $\mu_{0}$ (cf. Corollary 4). More specifically, if a $C^{\infty}$ system $\left(1_{\mu}\right)$ with $\boldsymbol{\mu} \in \mathbf{R}^{2}$ has a multiplicity-two limit cycle bifurcation curve $\mathscr{C}$ which terminates at a homoclinic loop bifurcation as $\boldsymbol{\mu} \rightarrow \boldsymbol{\mu}_{0} \equiv\left(\alpha_{0}, \beta_{0}\right)$, i.e., if as 

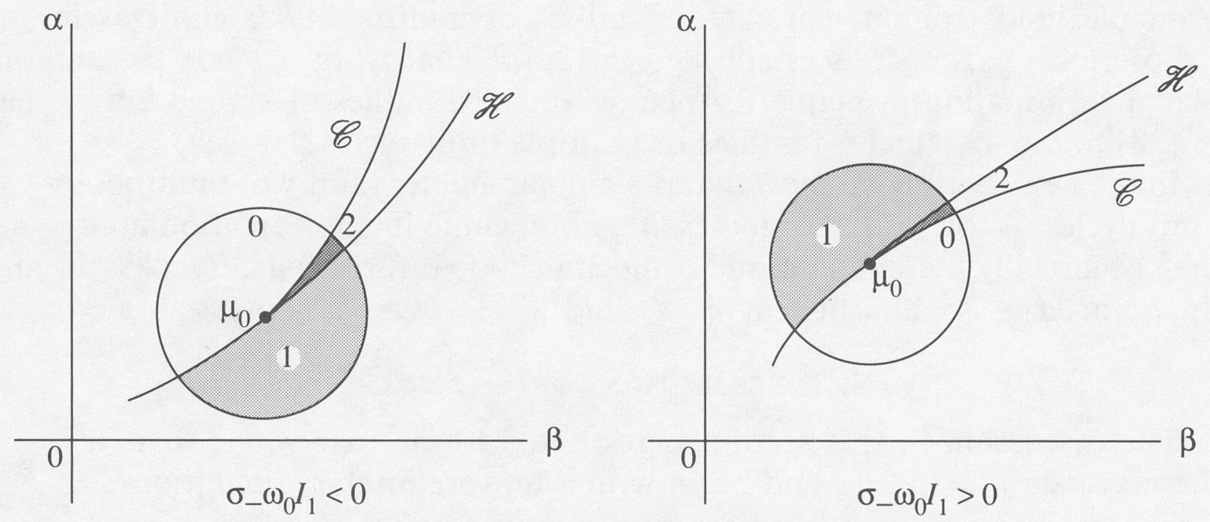

FIGURE 8. The bifurcation diagram for the system $\left(1_{\mu}\right)$ under the hypotheses of Theorem 3 where $\sigma_{-} \equiv \pm \operatorname{sgn} \nabla$ $\cdot \mathbf{f}(\mathbf{0}, h(\beta), \beta)$ for $\beta<\beta_{0}$ according to whether the Poincare map is defined on the exterior or on the interior of $\Gamma_{0}$ respectively

$\beta \rightarrow \beta_{0}^{+}$the one-parameter family of multiplicity-two limit cycles $\mathbf{x}_{\beta}(t)$ of $\left(1_{\mu}\right)$ approaches a separatrix cycle $S_{0}=\Gamma_{0} \cup\{\mathbf{0}\}$ at a hyperbolic saddle point $\mathbf{0}$ of $\left(1_{\boldsymbol{\mu}_{0}}\right)$, then $\nabla \cdot \mathbf{f}\left(\mathbf{0}, \boldsymbol{\mu}_{0}\right)=0$. (Cf. Theorem 3.) Hence, the resonance condition (1.9) in [16] is satisfied and according to Theorem A in [16], we typically have a unique homoclinic loop bifurcation curve $\mathscr{H}$ through the point $\boldsymbol{\mu}_{0} \in \mathbf{R}^{2}$ (cf. Theorem 4) and furthermore, $\mathscr{H}$ and $\mathscr{C}$ intersect at a flat contact at $\boldsymbol{\mu}_{0} \in \mathbf{R}^{2}$ (cf. Theorem 5).

The next theorem shows that if a multiplicity-two limit cycle bifurcation curve $\mathscr{C}$ intersects a homoclinic loop bifurcation curve $\mathscr{H}$ at a point $\boldsymbol{\mu}_{0} \in \mathbf{R}^{2}$, then $\nabla \cdot \mathbf{f}\left(\mathbf{0}, \boldsymbol{\mu}_{0}\right)=0$; i.e., in the terminology of [16], we have a homoclinic bifurcation at resonant eigenvalues. (Note that in this case, the eigenvalues of $D \mathbf{f}\left(\mathbf{0}, \boldsymbol{\mu}_{0}\right)$ are equal in magnitude and of opposite sign.) Theorem 3 and its corollary also determine the local bifurcation diagram and the local phase portraits for the system $\left(1_{\mu}\right)$ with $\boldsymbol{\mu}$ near $\boldsymbol{\mu}_{0}$ in the generic case. As on p. 183 in [16], a vector field $\mathbf{f} \in C^{\infty}\left(\mathbf{R}^{2} \times \mathbf{R}^{2}\right)$ is said to be generic if it belongs to a residual subset of $C^{\infty}\left(\mathbf{R}^{2} \times \mathbf{R}^{2}\right)$.

Theorem 3. In addition to the hypotheses of Theorem 1, suppose that there exists $a \delta>0$ such that the $C^{\infty}$ system $\left(1_{\mu}\right)$ with $\boldsymbol{\mu}=(\alpha, \beta) \in \mathbf{R}^{2}$ has $a$ homoclinic loop bifurcation curve $\mathscr{H}: \alpha=h(\beta)$ for $\beta_{0}-\delta<\beta<\beta_{0}+\delta$ and a multiplicity-two limit cycle bifurcation curve $\mathscr{C}: \alpha=g(\beta)$ for $\beta_{0}<\beta<$ $\beta_{0}+\delta$ which intersect at $\mu_{0}=\left(\alpha_{0}, \beta_{0}\right)$, i.e., $\alpha_{0}=h\left(\beta_{0}\right)=g\left(\beta_{0}^{+}\right)$and for all $t \in \mathbf{R}, \lim _{\beta \rightarrow \beta_{0}^{+}} \mathbf{x}_{\beta}(t) \in \Gamma_{0} \cup\{\mathbf{0}\}$, where $\mathbf{x}_{\beta}(t)$ is the one-parameter family of multiplicity-two limit cycles of $\left(1_{\mu_{\beta}}\right)$ with $\mu_{\beta}=(g(\beta), \beta)$, and $\Gamma_{0}$ is the homoclinic loop at the hyperbolic saddle point $\mathbf{0}$ of $\left(1_{\mu_{0}}\right)$. It then follows that $\nabla \cdot \mathbf{f}\left(\mathbf{0}, \boldsymbol{\mu}_{0}\right)=0$; and if the vector field $\mathbf{f} \in C^{\infty}\left(\mathbf{R}^{2} \times \mathbf{R}^{2}\right)$ is generic, it follows that $d[\nabla \cdot \mathbf{f}(\mathbf{0}, h(\beta), \beta)] / d \beta \neq 0$, i.e., $\nabla \cdot \mathbf{f}(\mathbf{0}, h(\beta), \beta)$ changes sign at $\beta=\beta_{0}$, and the bifurcation diagram for $\left(1_{\mu}\right)$ with $\boldsymbol{\mu}$ near $\boldsymbol{\mu}_{0}$ is given in Figure 8 where $I_{1}$ is defined by equation (2). 


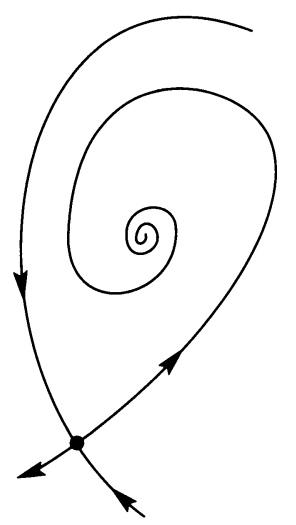

0

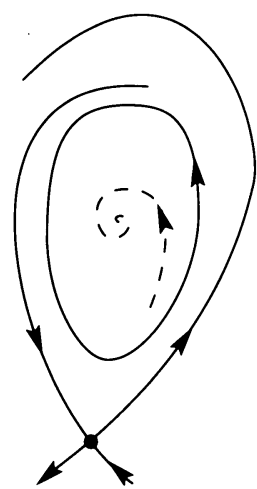

1

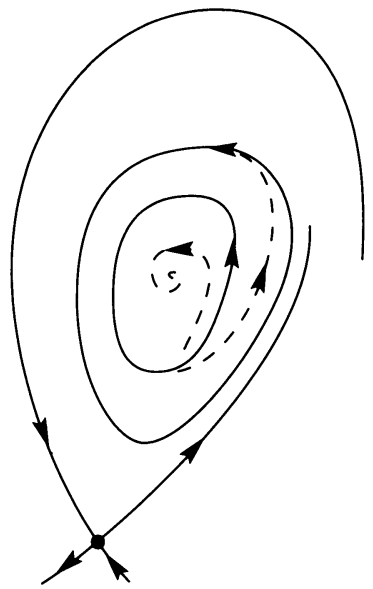

2

FIGURE 9. The local phase portraits for the system $\left(1_{\mu}\right)$ under the hypotheses of Corollary 3 with $\mu$ in the regions 0,1 , and 2 shown in Figure 8

Corollary 3. Under the hypotheses of Theorem 3, if $\omega_{0}>0, \sigma_{-}<0$, and the Poincare map is defined on the interior of $\Gamma_{0}$, then the phase portraits for the system $\left(1_{\mu}\right)$ with $\boldsymbol{\mu}$ in the regions 0,1 , and 2 in Figure 8 are given in Figure 9.

Remark 7. The phase portraits for the other cases that can occur in Corollary 3 are not difficult to determine (since the limit cycle that bifurcates from a simple separatrix cycle $\Gamma_{0}$ has the same stability as the separatrix cycle); e.g., if $\omega_{0}<0$ and $\sigma_{-}<0$ in Corollary 3 , then the phase portraits for the system $\left(1_{\mu}\right)$ are obtained simply by reversing the direction of the flow in Figure 9. More importantly, whether the one-parameter family of multiplicity-two limit cycles $\mathscr{C}$ in Figure 8 exists for $\beta>\beta_{0}$ or for $\beta<\beta_{0}$ is determined by the stability of the degenerate homoclinic loop $\Gamma_{0}$ of $\left(1_{\mu_{0}}\right)$ and, in particular by the sign of $I_{0}$, defined by (4); i.e., if $I_{0}<0$ then $\mathscr{C}$ exists for $\beta>\beta_{0}$ (as in Figure 8) and if $I_{0}>0$ then $\mathscr{C}$ exists for $\beta<\beta_{0}$ (as in Figure 8 rotated through $\pi$ radians about the line $\beta=\beta_{0}$ ).

Theorem 3 and its corollary follow directly from Theorems 1 and 2, Corollary 1 and the following lemma, which is Lemma 4 in [25].

Lemma 4. Under the hypotheses of Theorem 3,

$$
\lim _{\beta \rightarrow \beta_{0}^{+}} 2 J_{j}(\beta)=I_{j}
$$

for $j=1,2$ where $J_{j}(\beta)$ is defined by equation (14) with $\boldsymbol{\mu}=\boldsymbol{\mu}_{\beta} \equiv(g(\beta), \beta)$ and $g(\beta)$ is defined in the hypotheses of Theorem 3 .

We may, without loss of generality, assume that $\boldsymbol{\mu}_{0}=\mathbf{0}$; otherwise, we can translate the origin of the parameter space $\mathbf{R}^{2}$ to the point $\mu_{0}$. The next theorem is Theorem A, p. 183 in [16] for the case when $\mathbf{x} \in \mathbf{R}^{2}$.

Theorem 4. Suppose that $\mathbf{f}$ is a generic, $C^{\infty}$ vector field, $\mathbf{f}: \mathbf{R}^{2} \times \mathbf{R}^{2} \rightarrow \mathbf{R}^{2}$, that the system $\left(1_{\boldsymbol{\mu}}\right)$ with $\boldsymbol{\mu}=0$ has a homoclinic orbit $\Gamma_{0}$ at the hyperbolic saddle 
point $\mathbf{0}$ of $\left(1_{0}\right)$, and that the "resonance condition" $\nabla \cdot \mathbf{f}(\mathbf{0}, \mathbf{0})=0$ is satisfied. It then follows that there exists $a \delta>0$ and a $C^{\infty}$ diffeomorphic change of parameters $\boldsymbol{\epsilon}=\boldsymbol{\epsilon}(\boldsymbol{\mu})$ at $\boldsymbol{\mu}=\mathbf{0}$, with inverse $\boldsymbol{\mu}=\boldsymbol{\mu}(\boldsymbol{\epsilon})$, and a function $\kappa\left(\varepsilon_{1}\right)$ continuous on $[0, \delta)$ and of class $C^{\infty}$ on $(0, \delta)$ with $\kappa(0)=0$ such that $\left(1_{\mu}\right)$ has a unique, multiplicity-two limit cycle bifurcation curve $\mathscr{C}: \varepsilon_{2}=\kappa\left(\varepsilon_{1}\right)$ on $(0, \delta)$ and a unique homoclinic loop bifurcation curve $\mathscr{H}: \varepsilon_{2}=0$ on $(-\delta, \delta)$; furthermore, there exist constants $a_{0}>1$ and $k_{0}>0$ such that

$$
\lim _{\varepsilon_{1} \rightarrow 0^{+}}\left(\kappa\left(\varepsilon_{1}\right) a_{0}^{1 / \varepsilon_{1}} / \varepsilon_{1}\right)=k_{0}
$$

and if for $\varepsilon \equiv \varepsilon_{1} \in(0, \delta), \mathbf{x}_{\varepsilon}(t)$ denotes the one-parameter family of multiplicitytwo limit cycles of $\left(1_{\boldsymbol{\mu}}\right)$ with $\boldsymbol{\mu}=\boldsymbol{\mu}(\varepsilon, \kappa(\varepsilon))$, then for all $t \in \mathbf{R}, \lim _{\varepsilon \rightarrow 0^{+}} \mathbf{x}_{\varepsilon}(t) \in$ $\Gamma_{0} \cup\{\boldsymbol{0}\}$.

Theorem 5. Under the hypotheses of Theorem 4, the multiplicity-two limit cycle bifurcation curve $\mathscr{C}$ and the homoclinic loop bifurcation curve $\mathscr{H}$ have a flat contact at $\boldsymbol{\mu}=\mathbf{0}$.

Corollary 4. Under the hypotheses of Theorem 3, if $\mathbf{f}$ is a generic vector field, then $\mathscr{C}$ and $\mathscr{H}$ have a flat contact at $\boldsymbol{\mu}=\boldsymbol{\mu}_{0}$.

Theorem 5 follows from the next lemma, which implies that $\kappa\left(0^{+}\right)=\kappa^{\prime}\left(0^{+}\right)$ $=\kappa^{\prime \prime}\left(0^{+}\right)=\cdots=0$.

Lemma 5. Under the hypotheses of Theorem 4, for $n=0,1,2, \ldots$

$$
\kappa^{(n)}(\varepsilon)=O\left(a_{0}^{-1 / \varepsilon} / \varepsilon^{2 n-1}\right)
$$

as $\varepsilon \rightarrow 0^{+}$.

Before proving this lemma, we note that under the hypotheses of Theorem 4, it follows from Corollary 4.3, p. 205 in [16] that there exists a function $a(\epsilon)$ which is locally of class $C^{\infty}$ near $\epsilon=\mathbf{0}$ such that the bifurcation equation (4.8) on p. 203 in [16] has the normal form

$$
r=\varepsilon_{2}+a\left(\varepsilon_{1}, \varepsilon_{2}\right) r^{1+\varepsilon_{1}}+O\left(r^{1+\omega}\right)
$$

as $\varepsilon \rightarrow 0^{+}$where $0<\omega<1$; furthermore, according to Remark 4.4, p. 206 in [16], $a_{0} \equiv a(0)>1$ and, as in the proof of Lemma 5.3 on p. 211 in [16], for $r$ and $\varepsilon \equiv \varepsilon_{1}$ near zero, there exists a unique $C^{\infty}$ solution $\varepsilon_{2}(r, \varepsilon)$ of the "bifurcation equation"

$$
r=\varepsilon_{2}(r, \varepsilon)+a\left(\varepsilon, \varepsilon_{2}(r, \varepsilon)\right) r^{1+\varepsilon}+O\left(r^{1+\omega}\right)
$$

as $\varepsilon \rightarrow 0^{+}$where $\omega$ is some fixed constant with $0<\omega<1$; cf. equation (5.11a), p. 211 in [16]. Also, as on p. 211 in [16], we let $r_{*}(\varepsilon)$ be the unique $C^{\infty}$ solution of the equation defining the "fold condition" (along the curve $\mathscr{C}$ )

$$
1=(1+\varepsilon) a\left(\varepsilon, \varepsilon_{2}\left(r_{*}(\varepsilon), \varepsilon\right)\right) r_{*}(\varepsilon)^{\varepsilon}+O\left(r^{*}(\varepsilon)^{\omega}\right)
$$

as $\varepsilon \rightarrow 0^{+}$; cf. equation (5.11b), p. 211 in [16]. The next lemma then follows directly from Lemma 5.3 on p. 211 in [16] and the fact that $a\left(\varepsilon, \varepsilon_{2}\left(r_{*}(\varepsilon), \varepsilon\right)\right)=$ $a_{0}+a_{1} \varepsilon+O\left(\varepsilon^{2}\right)$ as $\varepsilon \rightarrow 0^{+}$which follows from Taylor's theorem. 
Lemma 6. Under the hypotheses of Theorem 4, there are constants $a_{0}>1$ and $k_{0}>0$ such that

$$
\lim _{\varepsilon \rightarrow 0^{+}} a_{0}^{1 / \varepsilon} r_{*}(\varepsilon)=k_{0}, \quad \lim _{\varepsilon \rightarrow 0^{+}} r_{*}(\varepsilon)^{\varepsilon}=1 / a_{0}, \quad \lim _{\varepsilon \rightarrow 0^{+}} \varepsilon \ln r_{*}(\varepsilon)=\ln 1 / a_{0},
$$

and

$$
\lim _{\varepsilon \rightarrow 0^{+}}\left(a_{0}^{1 / \varepsilon} \kappa(\varepsilon) / \varepsilon\right) \rightarrow k_{0}
$$

where $a_{0} \equiv a(\mathbf{0})>1, k_{0}=\exp \left[-1-a_{1} / a_{0}\right], \kappa(\varepsilon)$ is the function defined in Theorem 4 and $r_{*}(\varepsilon)$ is the solution of $(22)$.

Remark 8. It can be shown that

$$
r_{*}(\varepsilon)=k_{0} a_{0}^{-1 / \varepsilon}[1+O(\varepsilon)] \text { and } \kappa(\varepsilon)=k_{0} \varepsilon a_{0}^{-1 / \varepsilon}[1+O(\varepsilon)]
$$

as $\varepsilon \rightarrow 0^{+}$; however, this will not be necessary for our purpose.

Proof of Lemma 5. As in [16], let the functions $\varepsilon_{2}(r, \varepsilon)$ and $r_{*}(\varepsilon)$ be the unique, $C^{\infty}$ solutions of $(21)$ and (22) respectively and, as on p. 212 in [16], define the function $\kappa(\varepsilon) \equiv \varepsilon_{2}\left(r_{*}(\varepsilon), \varepsilon\right)$ ) for small $\varepsilon>0$ and define $\kappa(0) \equiv 0$. It then follows from Lemma 5.3 in [16] that $\kappa \in C[0, \delta)$ for some $\delta>0$ and it follows, as in the proof of Lemma 5.3 in [16], that for $0<\varepsilon<\delta, \kappa(\varepsilon)$ and $r_{*}(\varepsilon)$ are the unique $C^{\infty}$ solutions of

$$
\kappa(\varepsilon)=r_{*}(\varepsilon)-a(\varepsilon, \kappa(\varepsilon)) r_{*}(\varepsilon)^{1+\varepsilon}+O\left(r_{*}(\varepsilon)^{1+\omega}\right)
$$

and

$$
1=(1+\varepsilon) a(\varepsilon, \kappa(\varepsilon)) r_{*}(\varepsilon)^{\varepsilon}+O\left(r_{*}(\varepsilon)^{\omega}\right)
$$

as $\varepsilon \rightarrow 0^{+}$respectively. The first of these equations could be used to obtain asymptotic estimates for $\kappa^{(n)}(\varepsilon)$ in terms of $r_{*}^{(n)}(\varepsilon)$; however, a sharper estimate can be obtained if we substitute equation (23) into the above equation for $\kappa(\varepsilon)$ to obtain

$$
\kappa(\varepsilon)=\varepsilon a(\varepsilon, \kappa(\varepsilon)) r_{*}(\varepsilon)^{1+\varepsilon}+O\left(r_{*}(\varepsilon)^{1+\omega}\right)
$$

as $\varepsilon \rightarrow 0^{+}$.

If we let $D \equiv d / d \varepsilon$, it then follows from (23) and the fact, established in Lemma 4.2, p. 203 in [16], that the error terms in (23) and (24) can be differentiated any number of times, that

$$
\begin{aligned}
(1+\varepsilon) & a(\varepsilon, \kappa(\varepsilon)) D\left[r_{*}(\varepsilon)^{\varepsilon}\right]=-D[(1+\varepsilon) a(\varepsilon, \kappa(\varepsilon))] r_{*}(\varepsilon)^{\varepsilon}+O\left(D\left[r_{*}(\varepsilon)^{\omega}\right]\right) \\
= & -\left[a(\varepsilon, \kappa(\varepsilon))+(1+\varepsilon) \frac{\partial a}{\partial \varepsilon_{1}}(\varepsilon, \kappa(\varepsilon))+(1+\varepsilon) \frac{\partial a}{\partial \varepsilon_{2}}(\varepsilon, \kappa(\varepsilon)) \kappa^{\prime}(\varepsilon)\right] r_{*}(\varepsilon)^{\varepsilon} \\
& +O\left[r_{*}(\varepsilon)^{\omega-1} r_{*}^{\prime}(\varepsilon)\right]
\end{aligned}
$$

as $\varepsilon \rightarrow 0^{+}$. And then from (24) and Lemma 6,

$$
\begin{aligned}
\kappa^{\prime}(\varepsilon)= & \varepsilon a(\varepsilon, \kappa(\varepsilon)) r_{*}(\varepsilon)^{\varepsilon} r_{*}^{\prime}(\varepsilon) \\
& +\left[a(\varepsilon, \kappa(\varepsilon))+\varepsilon \frac{\partial a}{\partial \varepsilon_{1}}(\varepsilon, \kappa(\varepsilon))+\varepsilon \frac{\partial a}{\partial \varepsilon_{2}}(\varepsilon, \kappa(\varepsilon)) \kappa^{\prime}(\varepsilon)\right] r_{*}(\varepsilon)^{1+\varepsilon} \\
& +O\left(D\left[r_{*}(\varepsilon)^{1+\omega}\right]\right)
\end{aligned}
$$

which, in turn, implies that

$$
\kappa^{\prime}(\varepsilon)=\varepsilon a_{0} r_{*}(\varepsilon)^{\varepsilon} r_{*}^{\prime}(\varepsilon)[1+O(\varepsilon)]
$$


as $\varepsilon \rightarrow 0^{+}$. Substituting (25) into the equation above for $D\left[r_{*}(\varepsilon)^{\varepsilon}\right]$ and using Lemma 6 then leads to

$$
D\left[r_{*}(\varepsilon)^{\varepsilon}\right]=O(1)+O\left(r_{*}(\varepsilon)^{\omega-1} r_{*}^{\prime}(\varepsilon), \varepsilon r_{*}^{\prime}(\varepsilon)\right)
$$

or

$$
\varepsilon \frac{r_{*}^{\prime}(\varepsilon)}{r_{*}(\varepsilon)}+\ln r_{*}(\varepsilon)=O(1)+O\left(r_{*}(\varepsilon)^{\omega-1} r_{*}^{\prime}(\varepsilon), \varepsilon r_{*}^{\prime}(\varepsilon)\right)
$$

or

$$
r_{*}^{\prime}(\varepsilon)\left[1+O\left(r_{*}(\varepsilon)^{\omega} / \varepsilon, r_{*}(\varepsilon)\right)\right]=\frac{-r_{*}(\varepsilon) \ln r_{*}(\varepsilon)}{\varepsilon}[1+O(\varepsilon)]
$$

or finally

$$
r_{*}^{\prime}(\varepsilon)=\frac{-r_{*}(\varepsilon) \ln r_{*}(\varepsilon)}{\varepsilon}[1+O(\varepsilon)]
$$

as $\varepsilon \rightarrow 0^{+}$since $r_{*}(\varepsilon)^{\omega} / \varepsilon=O(\varepsilon)$ and $r_{*}(\varepsilon)=O(\varepsilon)$ as $\varepsilon \rightarrow 0^{+}$according to Lemma 6. It follows, using Lemma 6, that

$$
\lim _{\varepsilon \rightarrow 0^{+}} \varepsilon^{2} a_{0}^{1 / \varepsilon} r_{*}^{\prime}(\varepsilon)=k_{0} \ln a_{0}
$$

It then follows from Lemma 6, (25), and (27) that

$$
\lim _{\varepsilon \rightarrow 0^{+}} \varepsilon a_{0}^{1 / \varepsilon} \kappa^{\prime}(\varepsilon)=k_{0} \ln a_{0} \text {. }
$$

Next, it follows from (23) that

$$
\begin{aligned}
(1+\varepsilon) & a(\varepsilon, \kappa(\varepsilon)) D^{2}\left[r_{*}(\varepsilon)^{\varepsilon}\right] \\
= & -D^{2}[(1+\varepsilon) a(\varepsilon, \kappa(\varepsilon))] r_{*}(\varepsilon)^{\varepsilon}-2 D[(1+\varepsilon) a(\varepsilon, \kappa(\varepsilon))] D\left[r_{*}(\varepsilon)^{\varepsilon}\right] \\
& +O\left(D^{2}\left[r_{*}(\varepsilon)^{\omega}\right]\right)
\end{aligned}
$$

and from (24) that

$$
\begin{aligned}
\kappa^{\prime \prime}(\varepsilon)= & \varepsilon a(\varepsilon, \kappa(\varepsilon)) D^{2}\left[r_{*}(\varepsilon)^{1+\varepsilon}\right]+2 D[\varepsilon a(\varepsilon, \kappa(\varepsilon))] D\left[r_{*}(\varepsilon)^{1+\varepsilon}\right] \\
& +D^{2}[\varepsilon a(\varepsilon, \kappa(\varepsilon))] r_{*}(\varepsilon)^{1+\varepsilon}+O\left(D^{2}\left[r_{*}(\varepsilon)^{1+\omega}\right]\right)
\end{aligned}
$$

which implies that

$$
\kappa^{\prime \prime}(\varepsilon)=\varepsilon a_{0} r_{*}(\varepsilon)^{\varepsilon} r_{*}^{\prime \prime}(\varepsilon)[1+O(\varepsilon)]
$$

as $\varepsilon \rightarrow 0^{+}$. Substituting (30) into the above equation for $D^{2}\left[r_{*}(\varepsilon)^{\varepsilon}\right]$, using (28), (29), and Lemma 6, then leads to

$$
D^{2}\left[r_{*}(\varepsilon)^{\varepsilon}\right]=O\left(1 / \varepsilon^{2}\right)+O\left(r_{*}(\varepsilon)^{\omega-1} r_{*}^{\prime \prime}(\varepsilon), \varepsilon r_{*}^{\prime \prime}(\varepsilon)\right)
$$

or

or

$$
\frac{\varepsilon r_{*}^{\prime \prime}(\varepsilon)}{r_{*}(\varepsilon)}-\frac{\varepsilon r_{*}^{\prime}(\varepsilon)^{2}}{r_{*}(\varepsilon)^{2}}=O\left(\frac{1}{\varepsilon_{2}}\right)+O\left(r_{*}(\varepsilon)^{\omega-1} r_{*}^{\prime \prime}(\varepsilon), \varepsilon r_{*}^{\prime \prime}(\varepsilon)\right)
$$

or finally

$$
r_{*}^{\prime \prime}(\varepsilon)\left[1+O\left(r_{*}(\varepsilon)^{\omega} / \varepsilon, r_{*}(\varepsilon)\right)\right]=\frac{r_{*}^{\prime}(\varepsilon)^{2}}{r_{*}(\varepsilon)}[1+O(\varepsilon)]
$$

$$
r_{*}^{\prime \prime}(\varepsilon)=\frac{r_{*}^{\prime}(\varepsilon)^{2}}{r_{*}(\varepsilon)}[1+O(\varepsilon)]
$$


as $\varepsilon \rightarrow 0^{+}$. It then follows from (27) and Lemma 6 that

$$
\lim _{\varepsilon \rightarrow 0^{+}} \varepsilon^{4} a_{0}^{1 / \varepsilon} r_{*}^{\prime \prime}(\varepsilon)=k_{0}\left(\ln a_{0}\right)^{2} .
$$

Thus, from (30) and (33),

$$
\lim _{\varepsilon \rightarrow 0^{+}} \varepsilon^{3} a_{0}^{1 /, \varepsilon} \kappa^{\prime \prime}(\varepsilon)=k_{0}\left(\ln a_{0}\right)^{2} .
$$

Proceeding in this manner, we assume that

$$
r_{*}^{(k)}(\varepsilon)=O\left(a_{0}^{-1 / \varepsilon} / \varepsilon^{2 k}\right) \text { and } \kappa^{(k)}(\varepsilon)=O\left(a_{0}^{-1 / \varepsilon} / \varepsilon^{2 k-1}\right)
$$

as $\varepsilon \rightarrow 0^{+}$for $k=0, \ldots, n-1$ and show, by induction, that these estimates hold for $k=n$. Using Lemma 6, it follows from (24) and the above assumptions that

$$
\kappa^{(n)}(\varepsilon)=\varepsilon a(\varepsilon, \kappa(\varepsilon)) D^{n}\left[r_{*}(\varepsilon)^{1+\varepsilon}\right][1+O(\varepsilon)]+O\left(D^{n}\left[r_{*}(\varepsilon)^{1+\omega}\right]\right)
$$

which implies that $k^{(n)}(\varepsilon)=O\left(\varepsilon r_{*}^{(n)}(\varepsilon)\right)$ as $\varepsilon \rightarrow 0^{+}$and then (23) implies that

$$
D^{n}\left[r_{*}(\varepsilon)^{\varepsilon}\right]=O\left(1 / \varepsilon^{2 n-2}\right)+O\left(r_{*}(\varepsilon)^{\omega-1} r_{*}^{(n)}(\varepsilon), \varepsilon r_{*}^{(n)}(\varepsilon)\right)
$$

which, after some lengthy algebraic simplification using Lemma 6, implies that

$$
r_{*}^{(n)}(\varepsilon)=O\left(r_{*}^{\prime}(\varepsilon)^{n} / r_{*}(\varepsilon)^{n-1}\right)=O\left(a_{0}^{-1 / \varepsilon} / \varepsilon^{2 n}\right)
$$

and this, in turn, implies that

$$
\kappa^{(n)}(\varepsilon)=O\left(a_{0}^{-1 / \varepsilon} / \varepsilon^{2 n-1}\right)
$$

as $\varepsilon \rightarrow 0^{+}$. This completes the proof of Lemma 5 .

Remark 9. The asymptotic estimate

$$
\kappa^{(n)}(\varepsilon)=O\left(a_{0}^{-1 / \varepsilon} / \varepsilon^{2 n-1}\right)
$$

as $\varepsilon \rightarrow 0^{+}$is just what one would expect from the result

$$
\kappa(\varepsilon)=O\left(\varepsilon a_{0}^{-1 / \varepsilon}\right)
$$

as $\varepsilon \rightarrow 0^{+}$in Theorem $\mathrm{A}$ in [16] since the $n$th derivative of the function $F(\varepsilon) \equiv \varepsilon a_{0}^{-1 / \varepsilon}$,

as $\varepsilon \rightarrow 0^{+} ;$in fact

$$
F^{(n)}(\varepsilon)=O\left(a_{0}^{-1 / \varepsilon} / \varepsilon^{2 n-1}\right)
$$

$$
F^{(n)}(\varepsilon)=\left(a_{0}^{-1 / \varepsilon} / \varepsilon^{2 n-1}\right)\left(\ln a_{0}\right)^{n}[1+O(\varepsilon)]
$$

as $\varepsilon \rightarrow 0^{+}$for $n=0,1,2, \ldots$.

Remark 10. In Theorem A of [16], i.e., Theorem 4, Chow, Deng, and Fiedler show that if the "resonance condition" $\nabla \cdot \mathbf{f}\left(\mathbf{0}, \boldsymbol{\mu}_{0}\right)=0$ is satisfied for a system $\left(1_{\mu_{0}}\right)$ having a homoclinic loop $\Gamma_{0}$ at a hyperbolic saddle point $\mathbf{0}$, then "resonant side switching" typically occurs at $\mu_{0}$; i.e., in the case when $\mathbf{f}$ is a generic vector field, there is a unique homoclinic loop bifurcation curve $\mathscr{H}$ through the point $\mu_{0}$ and a unique multiplicity-two limit cycle bifurcation curve $\mathscr{E}$, which has a flat contact with $\mathscr{H}$ at $\mu_{0}$ according to Theorems 4 and 5 . One disadvantage of these theorems is that it is usually difficult to establish that the vector field $\mathbf{f}$ is generic. However, in the case when $I_{1} \neq 0$ or when $\mathbf{f}(\mathbf{x}, \alpha, \beta)$ 


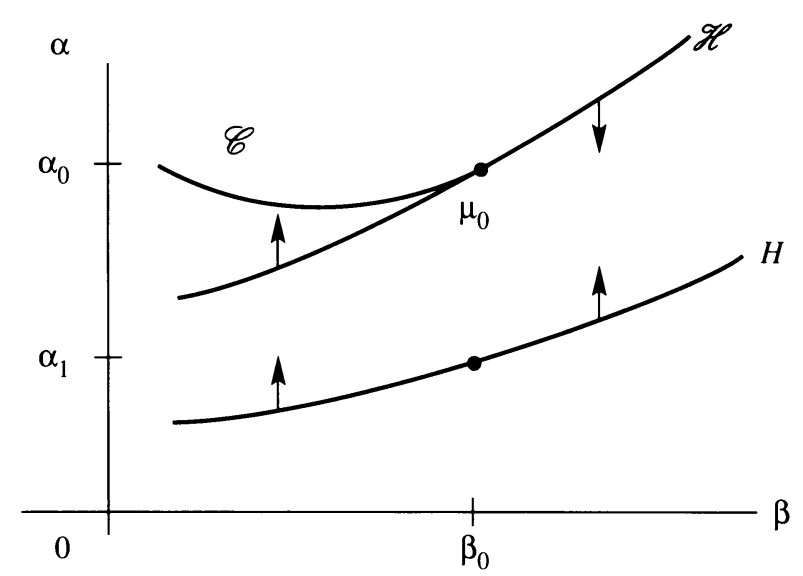

FIGURE 10. Resonant side switching at $\mu_{0}$ for the case when $\sigma_{0}(\beta) \sigma>0$ for $\beta<\beta_{0}$. The arrows indicate the side of $\mathscr{H}$ or $H$ on which a limit cycle bifurcates from $\Gamma_{0}$ or $\mathbf{x}_{1}$ respectively

defines a one-parameter family of rotated vector fields with parameter $\alpha$, the following set of conditions, given in terms of quantities which can be easily calculated, are sufficient for resonant side switching to occur at $\boldsymbol{\mu}_{0}=\left(\alpha_{0}, \beta_{0}\right)$ :

(1) Suppose that $\left(1_{\mu_{0}}\right)$ has a homoclinic loop $\Gamma_{0}$ at a hyperbolic saddle point $\mathbf{0}$, that $\nabla \cdot \mathbf{f}\left(\mathbf{0}, \boldsymbol{\mu}_{0}\right)=0$, and that $\nabla \cdot \mathbf{f}(\mathbf{0}, h(\beta), \beta)$ changes its sign at $\beta=\beta_{0}$, where the function $h(\beta)$ is defined in Theorem 1 .

(2) Suppose that for $\boldsymbol{\mu}=\left(\alpha_{1}, \beta_{0}\right)$ the system $\left(1_{\mu}\right)$ has a weak focus of multiplicity one at the critical point $\mathbf{x}_{1}$; i.e., $\nabla \cdot \mathbf{f}\left(\mathbf{x}_{1}, \alpha_{1}, \beta_{0}\right)=0$ and the first focal value $\sigma$ for $\left(1_{\mu}\right)$, defined by equation $\left(3^{\prime}\right)$ on p. 317 in [9], is not zero; and suppose that $(\partial / \partial \alpha) \nabla \cdot \mathbf{f}\left(\mathbf{x}_{1}, \alpha_{1}, \beta_{0}\right) \neq 0$. (It then follows that there is a unique Hopf bifurcation curve $\alpha=H(\beta)$ through the point $\left(\alpha_{1}, \beta_{0}\right)$ in the $(\alpha, \beta)$ plane.)

(3) Finally, suppose that $\mathbf{x}_{1}$ is the only critical point of $\left(1_{\mu}\right)$ on the interior of $\Gamma_{0}$ and that $\sigma_{1} \equiv\left(\alpha_{0}-\alpha_{1}\right) \omega_{0} \sigma I_{1}>0$, where $\omega_{0}$ is defined by the orientation of the homoclinic loop $\Gamma_{0}$ (or of the weak focus $\mathbf{x}_{1}$ ), $\sigma$ is defined by $\left(3^{\prime}\right)$ on p. 317 in [9], and $I_{1}$ is defined by (2). (This implies that the homoclinic loop bifurcation curve $\mathscr{H}$ lies on the side of the Hopf bifurcation curve $H$ on which there exists a limit cycle; cf. Figure 10.)

The theory of rotated vector fields in [7] or [17] can then be used to show that, under conditions (1)-(3) above, there exists a multiplicity-two limit cycle bifurcation curve $\mathscr{C}: \alpha=g(\beta)$ defined on the side of $\mu_{0}=\left(\alpha_{0}, \beta_{0}\right)$ where $\sigma_{0}(\beta) \sigma>0$, where $\sigma_{0}(\beta) \equiv \pm \nabla \cdot \mathbf{f}(\mathbf{0}, h(\beta), \beta)$ according to whether the Poincare map is defined on the exterior or interior of $\Gamma_{0}$ respectively, and $\lim _{\beta \rightarrow \beta_{0}} g(\beta)=\alpha_{0}$. Cf. Figure 10 for the case when $\sigma_{0}(\beta) \sigma>0$ for $\beta<\beta_{0}$.

There is another type of side switching that typically occurs when the system $\left(1_{\mu_{0}}\right)$ has a weak focus of multiplicity two. Since this occurs in many important examples in the literature, cf., e.g., [11-15], we give a set of conditions which are sufficient for this type of side switching to occur at $\boldsymbol{\mu}_{0}=\left(\alpha_{0}, \beta_{0}\right)$ when $I_{1} \neq 0$ : 


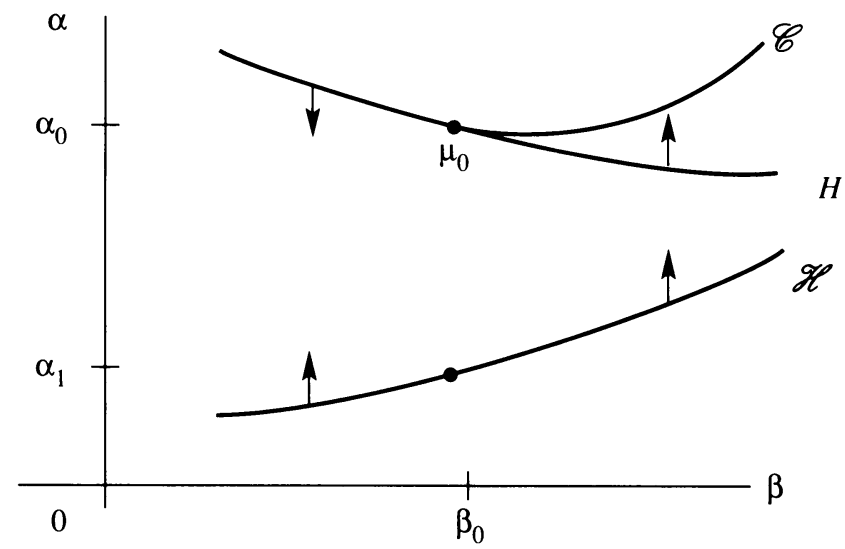

FIGURE 11. Side switching at $\mu_{0}$ for the case when $\sigma(\beta) \sigma_{0}>0$ for $\beta>\beta_{0}$. The arrows indicate the side of $H$ or $\mathscr{H}$ on which a limit cycle bifurcates from $\mathbf{x}_{0}$ or $\Gamma_{0}$ respectively

(1) Suppose that $\left(1_{\mu_{0}}\right)$ has a weak focus of multiplicity-two at the critical point $\mathbf{x}_{0}$ and that $(\partial / \partial \alpha) \nabla \cdot \mathbf{f}\left(\mathbf{x}_{0}, \alpha_{0}, \beta_{0}\right) \neq 0$. (It then follows that there is a unique Hopf bifurcation curve $\alpha=H(\beta)$ through the point $\left.\boldsymbol{\mu}_{0}.\right)$

(2) Suppose that for $\mu=\left(\alpha_{1}, \beta_{0}\right)$ the system $\left(1_{\mu}\right)$ has a homoclinic loop $\Gamma_{0}$ at a hyperbolic saddle point $\mathbf{0}$ and that $\nabla \cdot \mathbf{f}\left(\mathbf{0}, \alpha_{1}, \beta_{0}\right) \neq 0$.

(3) Suppose that $\mathbf{x}_{0}$ is the only critical point of $\left(1_{\mu}\right)$ on the interior of $\Gamma_{0}$ and that $\sigma_{1} \equiv\left(\alpha_{0}-\alpha_{1}\right) \omega_{0} \sigma_{0} I_{1}>0$, where $\omega_{0}$ is defined by the orientation of the homoclinic loop $\Gamma_{0}$ (or the weak focus $\mathbf{x}_{0}$ ), $\sigma_{0} \equiv$ $\pm \nabla \cdot \mathbf{f}\left(\mathbf{0}, \alpha_{1}, \beta_{0}\right)$ according to whether the Poincare map is defined on the exterior or interior of $\Gamma_{0}$ respectively, and $I_{1}$ is defined by (2). (This implies that the Hopf bifurcation curve $H$ lies on the side of the homoclinic loop bifurcation curve $\mathscr{H}$ on which there exists a limit cycle; cf. Figure 11.

The theory of rotated vector fields in [7] or [17] can then be used to show that there exists a multiplicity-two limit cycle bifurcation curve $\mathscr{C}: \alpha=g(\beta)$ defined on the side of $\mu_{0}=\left(\alpha_{0}, \beta_{0}\right)$ on which $\sigma_{(\beta)} \sigma_{0}>0$, where $\sigma_{(\beta)}$ is the first focal value for $\left(1_{\mu}\right)$, defined by $\left(3^{\prime}\right)$ on p. 317 in [9], and $\lim _{\beta \rightarrow \beta_{0}} g(\beta)=\alpha_{0}$. Cf. Figure 11 for the case when $\sigma(\beta) \sigma_{0}>0$ for $\beta>\beta_{0}$.

When both of the above types of side switching occur in a system $\left(1_{\mu}\right)$, we have "double side switching" that occurs as in the example of Rousseau at the end of $\S 2$. We end this paper with a set of conditions sufficient for double side switching to occur when $I_{1} \neq 0$.

(1) Suppose that $\left(1_{\mu_{0}}\right)$ has a homoclinic loop $\Gamma_{0}$ at a hyperbolic saddle point $\mathbf{0}$, that $\nabla \cdot \mathbf{f}\left(\mathbf{0}, \boldsymbol{\mu}_{0}\right)=0$, and that $\nabla \cdot \mathbf{f}(\mathbf{0}, h(\beta), \boldsymbol{\beta})$ changes its sign at $\beta=\beta_{0}$, where the function $h(\beta)$ is defined in Theorem 1 .

(2) Suppose that for $\mu_{1}=\left(\alpha_{1}, \beta_{1}\right)$, the system $\left(1_{\mu_{1}}\right)$ has a weak focus of multiplicity-two at the critical point $\mathbf{x}_{1}$ and that $(\partial / \partial \alpha) \nabla \cdot \mathbf{f}\left(\mathbf{x}_{1}, \boldsymbol{\mu}_{1}\right) \neq$ 0 . (It then follows that there is a unique Hopf bifurcation curve $\alpha=$ $H(\beta)$ through the point $\boldsymbol{\mu}_{1}$.) 


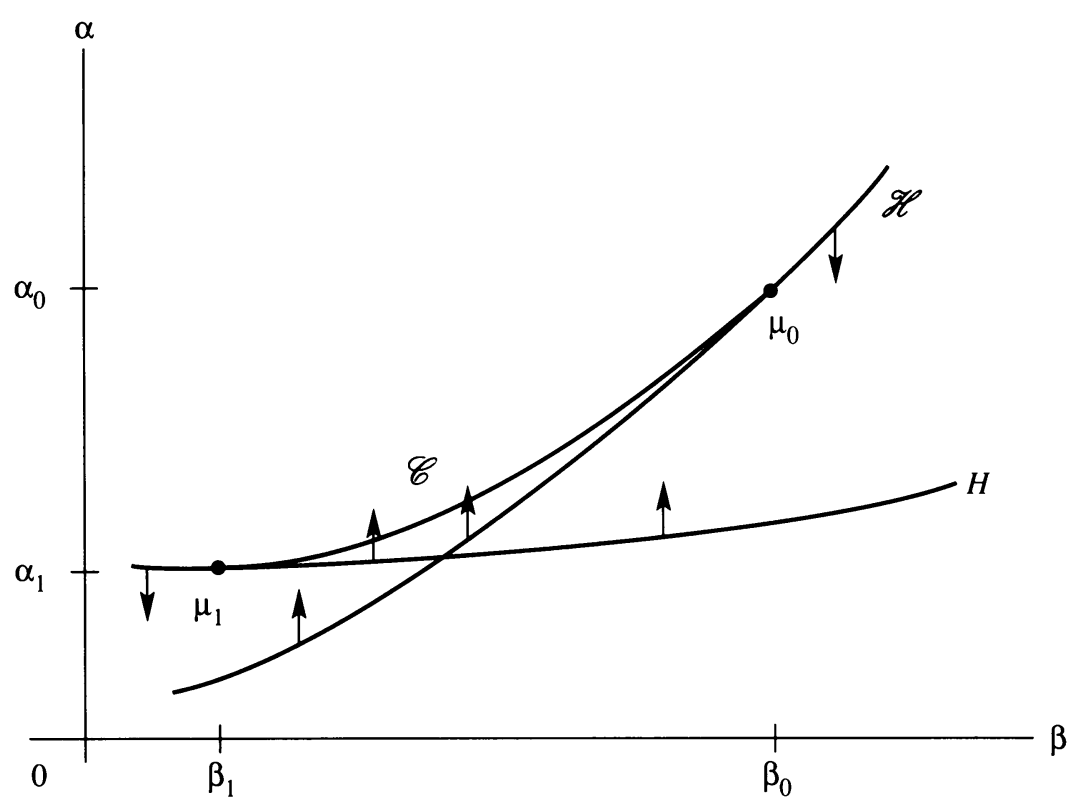

FIgURE 12. Double side switching at $\mu_{0}$ and $\mu_{1}$. The arrows indicate the side of $\mathscr{H}$ or $H$ on which a limit cycle bifurcates from $\Gamma_{0}$ or $\mathbf{x}_{1}$ respectively

(3) Suppose that $\mathbf{x}_{1}$ is the only critical point of $\left(1_{\mu}\right)$ on the interior of $\Gamma_{0}$, that $\beta_{1}<\beta_{0}$ and that for $\mu=\left(H\left(\beta_{0}\right), \beta_{0}\right), \sigma_{0} \equiv\left[\alpha_{0}-H\left(\beta_{0}\right)\right] \omega_{0} \sigma_{\left(\beta_{0}\right)} I_{1}$ $>0$, where $\sigma_{(\beta)}$ is defined for $\left(1_{\mu}\right)$ by $\left(3^{\prime}\right)$ on p. 317 in [9], and for $\boldsymbol{\mu}=\left(h\left(\beta_{1}\right), \beta_{1}\right), \sigma_{1} \equiv\left[\alpha_{1}-h\left(\beta_{1}\right)\right] \omega_{0} \sigma_{0}\left(\beta_{1}\right) I_{1}>0$, where $\sigma_{0}(\beta) \equiv$ $\pm \nabla \cdot \mathbf{f}(\mathbf{0}, h(\beta) \beta)$ according to whether the Poincare map is defined in the exterior or interior of $\Gamma_{0}$ respectively. Finally, suppose that $\sigma(\beta) \sigma_{0}(\beta)>0$ for $\beta_{1}<\beta<\beta_{0}$.

The theory of rotated vector fields in [7] or [17] can then be used to show that there exists a multiplicity-two limit cycle bifurcation curve $\mathscr{C}: \alpha=g(\beta)$ defined for $\beta_{1}<\beta<\beta_{0}$ with $\lim _{\beta \rightarrow \beta_{0}} g(\beta)=\alpha_{0}$ and $\lim _{\beta \rightarrow \beta_{1}} g(\beta)=\alpha_{1}$ and that we have the bifurcation diagram shown in Figure 12, or that bifurcation diagram rotated about the $\beta$-axis. The curve $\mathscr{C}$ has a flat contact with $\mathscr{H}$ at $\boldsymbol{\mu}_{0}$ and is tangent to $H$ at $\boldsymbol{\mu}_{1}$.

\section{APPENDIX I}

Under the hypotheses of Theorem 1 (with $k \geq 2$ ), we can obtain a result similar to the result in Theorem 1 for the system $\left(1_{\mu}\right)$ linearized about $\boldsymbol{\mu}=\boldsymbol{\mu}_{0}$,

$$
\begin{aligned}
\dot{\mathbf{x}} & =\mathbf{f}(\mathbf{x}, \boldsymbol{\mu})=\mathbf{f}\left(\mathbf{x}, \boldsymbol{\mu}_{0}\right)+\mathbf{f}_{\boldsymbol{\mu}}\left(\mathbf{x}, \boldsymbol{\mu}_{0}\right)\left(\boldsymbol{\mu}-\boldsymbol{\mu}_{0}\right)+O\left(\boldsymbol{\mu}-\boldsymbol{\mu}_{0}\right)^{2} \\
& =\mathbf{f}\left(\mathbf{x}, \boldsymbol{\mu}_{0}\right)+\varepsilon \mathbf{f}_{\boldsymbol{\mu}}\left(\mathbf{x}, \boldsymbol{\mu}_{0}\right) \boldsymbol{\nu}+O\left(\varepsilon^{2}\right) \\
& \equiv \mathbf{f}\left(\mathbf{x}, \boldsymbol{\mu}_{0}\right)+\varepsilon \mathbf{g}\left(\mathbf{x}, \boldsymbol{\mu}_{0}, \boldsymbol{\nu}\right)+O\left(\varepsilon^{2}\right)
\end{aligned}
$$

with $\boldsymbol{\mu}-\boldsymbol{\mu}_{0} \equiv \varepsilon \nu$ and $\mathbf{g}\left(\mathbf{x}, \boldsymbol{\mu}_{0}, \boldsymbol{\nu}\right) \equiv \mathbf{f}_{\boldsymbol{\mu}}\left(\mathbf{x}, \boldsymbol{\mu}_{0}\right) \boldsymbol{\nu}$, in terms of the Melnikov function $M\left(\mu_{0}, \nu\right)$ for the perturbed system (34),

$$
M\left(\boldsymbol{\mu}_{0}, \boldsymbol{\nu}\right) \equiv \int_{-\infty}^{\infty} \mathbf{e}^{-\int_{0}^{t} \nabla \cdot \mathbf{f}\left(\gamma_{0}\left(t^{\prime}\right), \boldsymbol{\mu}_{0}\right) d t^{\prime}} \mathbf{f}\left(\gamma_{0}(t), \boldsymbol{\mu}_{0}\right) \wedge \mathbf{g}\left(\gamma_{0}(t), \boldsymbol{\mu}_{0}, \boldsymbol{\nu}\right) d t
$$


given by equation (4.5.6), p. 187 in [8] or by equation (3), p. 379 in [9]. Since $\mathbf{g}_{v_{j}}\left(\mathbf{x}, \boldsymbol{\mu}_{0}, \mathbf{0}\right)=\mathbf{f}_{\mu_{j}}\left(\mathbf{x}, \boldsymbol{\mu}_{0}\right)$, it follows that

$$
M_{\nu_{j}}\left(\mu_{0}, \mathbf{0}\right)=\int_{-\infty}^{\infty} \mathbf{e}^{-\int_{0}^{t} \nabla \cdot \mathbf{f}\left(y_{0}\left(t^{\prime}\right), \boldsymbol{\mu}_{0}\right) d t^{\prime}} \mathbf{f} \wedge \mathbf{f}_{\mu_{j}}\left(\gamma_{0}(t), \boldsymbol{\mu}_{0}\right) d t .
$$

It then follows from Lemma 2, with the integrals $I_{j}$ given by equation (2), that

$$
d_{\mu_{j}}\left(\boldsymbol{\mu}_{0}\right)=-\frac{\omega_{0}}{\left|\mathbf{f}\left(\mathbf{x}_{0}, \boldsymbol{\mu}_{0}\right)\right|} M_{\nu_{j}}\left(\boldsymbol{\mu}_{0}, \mathbf{0}\right)=-\frac{\varepsilon \omega_{0}}{\left|\mathbf{f}\left(\mathbf{x}_{0}, \boldsymbol{\mu}_{0}\right)\right|} M_{\mu_{j}}\left(\boldsymbol{\mu}_{0}, \mathbf{0}\right) .
$$

This result was also obtained in [10]; cf. equation (15) in [10]. And from (35) we see that $d_{\varepsilon \mu_{j}}\left(\boldsymbol{\mu}_{0}\right)=-\omega_{0} M_{\mu_{j}}\left(\boldsymbol{\mu}_{0}, \mathbf{0}\right) /\left|\mathbf{f}\left(\mathbf{x}_{0}, \boldsymbol{\mu}_{0}\right)\right|$; cf. Lemma 1.1 in [6]. The relationship (35) between the displacement function for $\left(1_{\mu}\right)$ and the Melnikov function for (34) makes it clear that, under the hypotheses of Theorem 1 , we have $d\left(\mu_{0}\right)=0$ and $d_{\mu_{1}}\left(\mu_{0}\right) \neq 0$ for the system $\left(1_{\mu}\right)$ or equivalently $M\left(\mu_{0}, 0\right)=0$ and $M_{\nu_{j}}\left(\mu_{0}, 0\right) \neq 0$ for the perturbed system (34); thus, using the implicit function theorem, we can establish the existence of a family of homoclinic orbits for $\left(1_{\mu}\right)$, as in Theorem 1, or for (34), as in Theorem 3.1 in [6] or Theorem 4, p. 384 in [9] or Lemma 4 in [10].

Remark 11. Melnikov type integrals or equivalent computations appear in the 1890 paper [30] by H. Poincare (cf. the comments on p. 151 in [31]), in the 1953 paper [7] by G. F. D. Duff (cf. equation (3.1.7) in [7]), in the 1963 paper by V. K. Melnikov [32], in the 1964 thesis by J. Sotomayor [33], in the 1964 paper by V. I. Arnold [34], and in the book by A. A. Andronov et al. [1] published in 1966 (cf. equation (36), p. 384 in [1]; also see references [35] and [36]). It is therefore difficult to pin down the origins of Melnikov's method. Suffice it to say that the idea of computing the distance separating trajectories of $\left(1_{\mu}\right)$, i.e. computing the displacement function, as well as its partial derivatives with respect to parameters, in terms of certain integrals along the trajectories of $\left(1_{\mu}\right)$ has been used by many mathematicians working on the theory of nonlinear systems of ordinary differential equations at various times during the past 100 years.

\section{APPENDIX II}

In order to derive the variational equations of $\left(1_{\mu}\right)$ along a trajectory $\mathbf{x}(t, \mu)$ of $\left(1_{\mu}\right)$ near the homoclinic orbit $\Gamma_{0}$ of $\left(1_{\mu_{0}}\right)$, let $\omega_{0}$ denote the orientation of the separatrix cycle $S_{0}=\Gamma_{0} \cup\{\boldsymbol{0}\}$ and let $\alpha \equiv \mu_{1}$. Then for

$$
\boldsymbol{\xi}(t, \boldsymbol{\mu}) \equiv \partial \mathbf{x}(t, \boldsymbol{\mu}) / \partial \alpha
$$

we obtain the differential equation

$$
\dot{\boldsymbol{\xi}}=D \mathbf{f}(\mathbf{x}(t, \boldsymbol{\mu}), \boldsymbol{\mu}) \boldsymbol{\xi}+\mathbf{f}_{\alpha}(\mathbf{x}(t, \boldsymbol{\mu}), \boldsymbol{\mu})
$$

by differentiating $\left(1_{\mu}\right)$ with respect to $\alpha$ and interchanging the order of differentiation. In coordinates $(s, n)$ along a perpendicular to the trajectory $\mathbf{x}(t, \boldsymbol{\mu})$ of $\left(1_{\mu}\right)$ at the point $\mathbf{x}(0, \mu)$, the normal component of the first variation $\boldsymbol{\xi}(t, \boldsymbol{\mu})$ is given by

$$
\partial n / \partial \alpha=\omega_{0} \rho /|\mathbf{f}|
$$

where $\rho(t) \equiv \boldsymbol{\xi} \wedge \mathbf{f}(\mathbf{x}(t, \boldsymbol{\mu}), \boldsymbol{\mu})$; cf. Figure 13. This is equation (7) in $\S 2$ with $\mathbf{x}(t, \boldsymbol{\mu})=\mathbf{x}_{\boldsymbol{\mu}}^{s}(t)$. Since $\boldsymbol{\rho} \equiv \boldsymbol{\xi} \wedge \mathbf{f}=\boldsymbol{\xi} f_{2}-\xi_{2} f_{1}$, it follows that

$$
\dot{\rho}=\dot{\xi}_{1} f_{2}-\dot{\xi}_{2} f_{1}+\xi_{1} \dot{f}_{2}-\xi_{2} \dot{f}_{1} \text {. }
$$



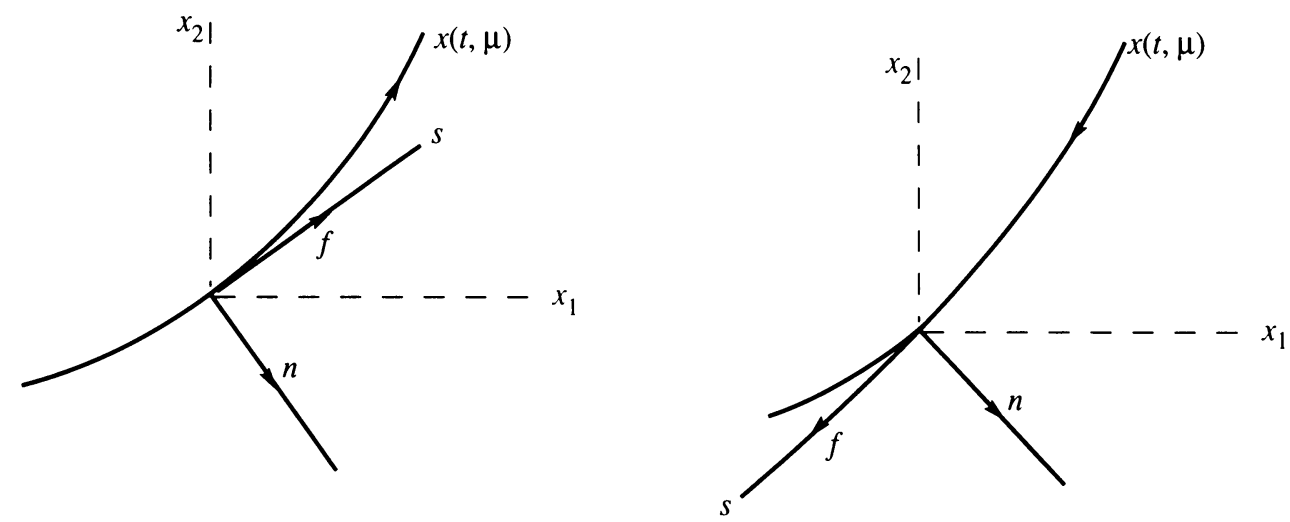

FIGURE 13. The local coordinates $(s, n)$ along and perpendicular to the trajectory $\mathbf{x}(t, \mu)$ of $\left(1_{\mu}\right)$ with $\mathbf{n}$ being the exterior normal

But, from the differential equation $\left(1_{\mu}\right)$, it follows that

$$
\dot{f}_{1}=\ddot{x}_{1}=\frac{\partial f_{1}}{\partial x_{1}} \dot{x}_{1}+\frac{\partial f_{1}}{\partial x_{2}} \dot{x}_{2}
$$

and

$$
\dot{f}_{2}=\ddot{x}_{2}=\frac{\partial f_{2}}{\partial x_{1}} \dot{x}_{1}+\frac{\partial f_{2}}{\partial x_{2}} \dot{x}_{2} .
$$

Substituting (36) and (38) into (37) then leads to

$$
\dot{\rho}=(\nabla \cdot \mathbf{f}) \rho-\mathbf{f} \wedge \mathbf{f}_{\alpha}
$$

which is equation (8) in $\S 2$ with $\mathbf{x}(t, \mu)=\mathbf{x}_{\mu}^{s}(t)$. See equation (3.12) on p. 22 in [7].

\section{REFERENCES}

1. A. A. Andronov, E. A. Leontovich, I. I. Gordon, and A. G. Maier, Theory of bifurcations of dynamical systems on a plane, Israel Program for Scientific Translations, Jerusalem, 1971.

2. C. Chicone and M. Jacobs, Bifurcation of limit cycles from quadratic isochrones, J. Differential Equations 91 (1991), 268-326.

3. C. Chicone, On bifurcation of limit cycles from centers, Lecture Notes in Math., vol. 1455, Springer-Verlag, Berlin and New York, 1990, pp. 20-43.

4. L. M. Perko, Bifurcation of limit cycles: geometric theory, Proc. Amer. Math. Soc. 114 (1992), 225-236.

5. __ Bifurcation of limit cycles, Lecture Notes in Math., vol. 1455, Springer-Verlag, Berlin and New York, 1990, pp. 315-333.

6. T. R. Blows and L. M. Perko, Bifurcation of limit cycles from centers and separatrix cycles, SIAM Rev. (to appear).

7. G. F. D. Duff, Limit cycles and rotated vector fields, Ann. of Math. (2) 67 (1953), 15-31.

8. J. Guckenheimer and P. Holmes, Nonlinear oscillations, dynamical systems, and bifurcations of vector fields, Appl. Math. Sci., vol. 42, Springer-Verlag, Berlin and New York, 1983.

9. L. M. Perko, Differential equations and dynamical systems, Texts in Appl. Math., vol 7, Springer-Verlag, Berlin and New York, 1991. 
10. __ A global analysis of the Bogdanov-Takens system, SIAM J. Appl Math. 52 (1992), 1172-1192.

11. J. A. Sanders and R. Cushman, Limit cycles in the Josephson equation, SIAM J. Math. Anal. 17 (1986), 495-511.

12. C. Rousseau, Example of a quadratic system with two cycles appearing in a homoclinic loop bifurcation, J. Differential Equations 66 (1987), 140-150.

13. Chengzhi $\mathrm{Li}$ and $\mathrm{C}$. Rousseau, $A$ system with three limit cycles appearing in a Hopf bifurcation and dying in a homoclinic bifurcation: The cusp of order four, J. Differential Equations 79 (1989), 132-167.

14. F. Dumortier and P. Fiddelaers, Quadratic models for generic local 3-parameter bifurcations on the plane, Trans. Amer. Math. Soc. 326 (1991), 101-126.

15. F. Dumortier, R. Roussarie, and J. Sotomayor, Generic 3-parameter families of vector fields on the plane, unfolding a singularity with nilpotent linear part: The cusp case of codimension three, Ergodic Theory Dynamical, Systems 7 (1987), 375-413.

16. S. N. Chow, B. Deng, and B. Fiedler, Homoclinic bifurcation at resonant eigenvalues, J. Dynamics Differential Equations 2 (1990) 177-244.

17. L. M. Perko, Rotated vector fields, J. Differential Equations 103 (1992), 127-145.

18. S. Bochner and W. T. Martin, Several complex variables, Princeton Univ. Press, Princeton, NJ, 1948.

19. Chengzhi Li, C. Rousseau, and Xian Wang, A simple proof for the unicity of the limit cycle in the Bogdanov-Takens system, Canad. Math. Bull. 33 (1990), 84-92.

20. L. Dingjun, H. Maoan, and Z. Deming, The uniqueness of limit cycles bifurcating from a homoclinic orbit, preprint, 1990.

21. M. W. Hirsch and S. Smale, Differential equations, dynamical systems, and linear algebra, Academic Press, San Diego, 1974.

22. J. Dieudonné, Foundations of modern analysis, Academic Press, San Diego, 1960.

23. E. A. Coddington and N. Levinson, Theory of ordinary differential equations, McGraw-Hill, New York, 1955.

24. L. M. Perko, Multiple limit cycle bifurcation surfaces and global families of multiple limit cycles, J. Differential Equations (submitted).

25. _ Homoclinic loop and semistable (multiplicity-2) limit cycle bifurcation surfaces I and II, N.A.U. Research Report, 1991.

26. M. Golubitsky and D. G. Schaeffer, Singularities and goups in bifurcation theory, Appl. Math. Sci., vol. 51, Springer-Verlag, Berlin and New York, 1985.

27. S. N. Chow and J. K. Hale, Methods of bifurcation theory, Springer-Verlag, Berlin and New York, 1982.

28. L. M. Perko, Global families of limit cycles of planar analytic systems, Trans. Amer. Math. Soc. 322 (1990), 627-656.

29. A. A. Andronov, E. A. Leontovich, I. I. Gordon, and A. G. Maier, Qualitative theory of second-order dynamic systems, Israel Program for Scientific Translations, Jerusalem, 1973.

30. H. Poincaré, Sur les équations de la dynamique et le problème des trois corps, Acta Math. 13 (1890), 1-270.

31. P. Holmes, Poincaré, celestial mechanics, dynamical systems theory and chaos, Phys. Rep. 193 (1990), 137-163.

32. V. K. Melnikov, On the stability of the center for time periodic perturbations, Trans. Moscow Math. Soc. 12 (1963), 1-57.

33. J. Sotomayor, Estabilidade estrutural de primeira ordem e variedades de Banach, Doctoral thesis, IMPA, Brazil, 1964.

34. V. I. Arnold, Instability of dynamical systems with several degrees of freedom, Soviet Math. Dokl. 5 (1964), 581-585. 
35. A. A.Andronov and L. S. Pontryagin, Structurally stable systems, Dokl. Akad. SSSR 14 (1937).

36. A. A. Andronov, A. A. Vitt, and S. E. Khaikin The theory of oscillations, Fizmatgiz, 1959.

37. R. Roussarie, On the number of limit cycles which appear by a perturbation of a separatrix loop of planar vector fields, Bol. Soc. Brasil Mat. 17 (1986), 67-101.

Department of Mathematics, Northern Arizona University, Flagstaff, Arizona 86011-5717 